\title{
The Westerbork Northern Sky Survey (WENSS)
}

\section{A 570 square degree Mini-Survey around the North Ecliptic Pole*}

\author{
R.B. Rengelink ${ }^{1}$, Y. Tang ${ }^{2}$, A.G. de Bruyn ${ }^{2,3}$, G.K. Miley ${ }^{1}$, M.N. Bremer ${ }^{1,4}$, H.J.A. Röttgering ${ }^{1,4,5}$, \\ and M.A.R. Bremer ${ }^{1,6}$ \\ 1 Leiden Observatory, Postbus 9513, 2300 RA Leiden, The Netherlands \\ 2 Netherlands Foundation for Research in Astronomy, Postbus 2, 7990 AA Dwingeloo, The Netherlands \\ 3 Kapteyn Astronomical Institute, Postbus 800, 9700 AV Groningen, The Netherlands \\ 4 Institute of Astronomy, Madingley Road, Cambridge, CB3 0HE, UK \\ 5 Mullard Radio Astronomy Observatory, Cavendish Laboratory, Madingley Road, Cambridge, CB3 0HE, UK \\ 6 Space Research Organization of the Netherlands, Sorbonnelaan 2, 3584 CA Utrecht, The Netherlands
}

Received May 30; accepted October 25, 1996

\begin{abstract}
The Westerbork Northern Sky Survey (WENSS) is a low-frequency radio survey that will cover the whole sky north of $\delta=30^{\circ}$ at a wavelength of $92 \mathrm{~cm}$ to a limiting flux density of approximately $18 \mathrm{mJy}\left(5 \sigma_{\mathrm{rms}}\right)$. This survey has a resolution of $54^{\prime \prime} \times 54^{\prime \prime} \operatorname{cosec} \delta$ and a positional accuracy for strong sources of $1.5^{\prime \prime}$. Here we present a source list comprising 11299 sources and maps of 120 extended sources for a 570 square degree region around the north ecliptic pole, the so-called mini-survey. We discuss the errors and reliability of the source parameters and the completeness of the survey.
\end{abstract}

Key words: surveys — radio continuum: general

\section{Introduction}

Large sky surveys are of fundamental importance to astronomy. They provide an overall description of the universe and the generic properties of its many different constituents. Furthermore, complete and unbiased surveys are an indispensable tool in creating well defined samples of particular objects.

In the radio regime there is a long history of ever more sensitive and accurate large sky surveys over a wide range of frequencies. Both the Westerbork Synthesis Radio Telescope (WSRT) and the Very Large Array (VLA) are currently dedicating substantial parts of their observing time to conducting new large-scale radio surveys. During

Send offprint requests to: R.B. Rengelink

* Table 6 is only available in electronic form at the CDS via anonymous ftp to cdsarc.u-strasbg.fr (130.79.128.5) or via http://cdsweb.u-strasbg.fr/Abstract.html the next few years, exploitation of these surveys should produce an enormous amount of new information about the radio universe.

The Westerbork Northern Sky Survey (WENSS) is a new low-frequency radio survey, designed to cover the whole sky north of declination $30^{\circ}$ at a wavelength of $92 \mathrm{~cm}(325 \mathrm{MHz})$, and about a quarter of this region, concentrated at high galactic latitudes, at a wavelength of $49 \mathrm{~cm}(609 \mathrm{MHz})$, to a limiting flux density of approximately $18 \mathrm{mJy}\left(5 \sigma_{\mathrm{rms}}\right)$ and $15 \mathrm{mJy}$ respectively. The products from WENSS are maps and source lists for all four Stokes parameters $(I, Q, U, V)$. Maps will be produced at a resolution (FWHM of the restoring beam) of $54^{\prime \prime} \times 54^{\prime \prime} \operatorname{cosec} \delta$ at $92 \mathrm{~cm}$ and $28^{\prime \prime} \times 28^{\prime \prime} \operatorname{cosec} \delta$ at $49 \mathrm{~cm}$. The positional accuracy for strong sources is $1.5^{\prime \prime}$ at both $92 \mathrm{~cm}$ and $49 \mathrm{~cm}$. WENSS will distribute its maps in a standard $6^{\circ} \times 6^{\circ}$ format. These maps we call frames.

To carry out this survey in a reasonable amount of time, WENSS utilizes the mosaicing capability of the WSRT. Exploiting this technique, a pattern of 80 evenly spaced fields is observed at regular intervals over several 12 hour syntheses with different array configurations. In this way it is possible to sufficiently sample the visibilities for all 80 fields and thus reconstruct a map of the sky that is many times larger than the field of view of the WSRT $\left(2.67^{\circ} \mathrm{HPBW}\right.$ at $\left.92 \mathrm{~cm}\right)$. These maps are referred to as mosaics.

WENSS is complemented by two other radio surveys with comparable beam-sizes, that are currently underway; the NRAO VLA Sky Survey (NVSS) at $1.4 \mathrm{GHz}$ (Condon et al. 1993) and the $151 \mathrm{MHz} 7 \mathrm{C}$ survey (McGilchrist et al. 1990; Lacy et al. 1994; Visser et al. 1995). These surveys will, when combined, provide well defined 
Table 1. Characteristics of recent major radio surveys of the northern sky, including the Westerbork Northern Sky Survey

\begin{tabular}{|c|c|c|c|c|c|c|c|c|}
\hline & \multicolumn{2}{|c|}{ WENSS } & $87 \mathrm{~GB}$ & NVSS & FIRST & $6 \mathrm{C}$ & $7 \mathrm{C}$ & $8 \mathrm{C}$ \\
\hline \multirow{2}{*}{$\begin{array}{l}\text { Frequency }(\mathrm{MHz}) \\
\text { Sky coverage }\end{array}$} & 609 & 325 & 4850 & 1400 & 1400 & 151 & 151 & 38 \\
\hline & \multicolumn{2}{|c|}{$|b|>20^{\circ b}>30^{\circ}$} & $0^{\circ}<\delta<75^{\circ}$ & $\delta>-40^{\circ}$ & $b>30^{\circ}$ & $\delta>30^{\circ a}$ & $\delta>20^{\circ}$ & $\delta>60^{\circ}$ \\
\hline Sky Area (sr) & 0.7 & 3.1 & 6.1 & 10.3 & 3.1 & 2.8 & 4 & 0.8 \\
\hline \multicolumn{9}{|l|}{$\left(5 \sigma_{\mathrm{rms}}, \mathrm{mJy}\right)$} \\
\hline Source density $\left(\mathrm{sr}^{-1}\right)$ & $310^{4}$ & $710^{4}$ & $10^{4}$ & $210^{5}$ & $310^{5}$ & $10^{4}$ & $310^{4}$ & $610^{3}$ \\
\hline Resolution & $\begin{array}{c}28^{\prime \prime} \times \\
28^{\prime \prime} \operatorname{cosec} \delta\end{array}$ & $\begin{array}{c}54^{\prime \prime} \times \\
54^{\prime \prime} \operatorname{cosec} \delta\end{array}$ & $3.7^{\prime} \times 3.3^{\prime}$ & $45^{\prime \prime}$ & $5^{\prime \prime}$ & $\begin{array}{c}4.2^{\prime} \times \\
4.2^{\prime} \operatorname{cosec} \delta\end{array}$ & $\begin{array}{c}70^{\prime \prime} \times \\
70^{\prime \prime} \operatorname{cosec} \delta\end{array}$ & $\begin{array}{c}4.5^{\prime} \times \\
4.5^{\prime} \operatorname{cosec} \delta\end{array}$ \\
\hline $\begin{array}{l}\text { Positional uncertainty } \\
\text { (strong sources) }\end{array}$ & \multicolumn{2}{|c|}{$1.5^{\prime \prime}$} & $10^{\prime \prime}$ & $1^{\prime \prime}$ & $0.1^{\prime \prime}$ & $5^{\prime \prime}-10^{\prime \prime}$ & $1^{\prime \prime}$ & $30^{\prime \prime}$ \\
\hline Polarization & \multicolumn{2}{|c|}{$I, Q, U, V$} & I & $I, Q, U$ & I & I & I & I \\
\hline References $^{c}$ & \multicolumn{2}{|c|}{1} & 2 & 3 & 4 & 5 & 6 & 7 \\
\hline
\end{tabular}

Notes: a) The $6 \mathrm{C}$ catalogue does not completely cover this area. b) The $49 \mathrm{~cm}$ survey will only cover about 0.7 sr of this area. c) References: 1) This paper, 2) Gregory et al. (1996), 3) Condon et al. (1993), 4) Becker et al. (1995), 5) Hales et al. (1993), and references therein 6) Visser et al. (1996), and references therein, 7) Rees (1990).

spectral information on an estimated $10^{5}$ radio-sources. The $4.85 \mathrm{GHz}$ NRAO-Greenbank survey (Condon et al. 1989; Becker et al. 1991; Gregory \& Condon 1991), recently updated (Gregory et al. 1995), the 6C survey at $151 \mathrm{MHz}$ (Hales et al. 1993), and the 8C survey at 38 $\mathrm{MHz}$ (Rees 1990; Lacy et al. 1992) provide additional information for a subset of these sources. The high resolution VLA survey at $21 \mathrm{~cm}$ of the north galactic cap, the Faint Images of the Radio Sky at Twenty cm (FIRST, Becker et al. 1995), will provide accurate positions and morphological information for a large number of WENSS sources in this region. Table 1 summarizes the characteristics of WENSS and several other major radio surveys.

The most important additional information that WENSS provides compared to previous radio surveys are:

- Radio spectral information on an unprecedented number of sources over a substantial fraction of the sky. WENSS will provide spectral information, both internally $(327 / 610 \mathrm{MHz})$, and by comparison with radio surveys at other frequencies. Thus WENSS will permit the study of very large numbers of sources with extreme spectra. These include: ultra steep spectrum (USS) sources (e.g. high-redshift radio galaxies, cluster halos, head-tail galaxies), peaked spectrum sources (e.g. GPS sources), and flat spectrum sources (e.g. high-redshift quasars)

- WENSS yields good positional information (from 5 $10^{\prime \prime}$ for the faintest sources to $1.5^{\prime \prime}$ for the brighter ones). For a large number of sources this is sufficient to obtain an optical identification.

- The sensitive polarization information coupled with the large number of sources give WENSS unique capa- bilities in searching for radio sources having (anomalously) high linear polarizations at low frequencies. These include pulsars as well as interesting variable extra galactic radio sources. The sensitivity to extended structure has made it possible to study the large scale distribution of diffuse polarized galactic foreground emission.

- Because of the large dynamic range of the WSRT, the good coverage of short baselines, and the relatively low resolution, WENSS will provide data on faint extended structures, ranging in sizes from $30^{\prime \prime}$ to $1^{\circ}$, over a large region of the sky. This will be particularly valuable for detecting large galactic and extra galactic radio sources.

- The mosaicing technique, used in constructing WENSS, requires several observations per field, and provides limited data on the low-frequency variability of a large number of sources over time-scales from hours to years.

\subsection{Outline}

In Sect. 2 of this paper we start with an introduction to the observational techniques used in producing WENSS. Included in this section are a discussion of the mosaicing concept (2.1), the observational set-up (2.2), the data reduction (2.3), the frame production (2.4), and the source extraction (2.5).

The first WENSS fields to be observed (in the spring of 1991) covered a 570 square degree area centered on the north ecliptic pole: the mini-survey. We present results for the total intensity data from this region. The minisurvey is described in Sect. 3. An overview of the region 
is presented (3.1), followed by a description of the source list (3.2). This source list is only available in electronic form. A correction to the measured flux densities of faint sources is discussed $(3.3,4)$, followed by a description of the errors (3.5) and the completeness (3.6).

Plots for a selection of sources characterized by an exceptional morphology (Appendix A) are given at the end of the paper.

\section{WENSS}

\subsection{Mosaicing}

Interferometry with an east-west array uses the Earth's rotation to sample visibilities over complete elliptical loci in the spatial frequency $(u, v)$ plane. 12 hours are normally required to image a single field. Given the small primary beam $\left(2.67^{\circ} \mathrm{HPBW}\right.$ at $\left.92 \mathrm{~cm}\right)$ of the WSRT, mapping of large areas of sky in this way is prohibitively time consuming. However, at the price of a decrease in sensitivity, a reasonable synthesized beam can be obtained by observing a single field for considerably less than these 12 hours, provided the visibilities are sampled for several short integrations, regularly spaced throughout a 12 hour observation. By cycling through a regular grid of pointings and observing each field intermittently, a relatively large area of sky can thus be mapped efficiently. This "mosaicing" technique was implemented as a standard observing mode at the WSRT in 1990 (Kolkman 1993) and has been used to construct WENSS.

WENSS utilizes mosaicing patterns of approximately 80 fields, covering about 100 square degrees. Using an integration time of 20 seconds and a slew time of 10 seconds this results in 18 "spokes" per field, per single 12 hour synthesis. For the $92 \mathrm{~cm}$ observations, six different telescope configurations are combined for a total of 108 spokes per field. The configurations are defined by the separation between telescope "9" and telescope "A", which are: 36, 48, $60,72,84$, and $96 \mathrm{~m}$. This results in a radial sampling of $12 \mathrm{~m}$, corresponding to half the antenna diameter. This sampling strategy results in a position for the first grating ring at $4.4^{\circ}$ in right ascension and $4.4^{\circ} \operatorname{cosec} \delta$ in declination. Figure 1 illustrates the $u, v$-sampling of a single field for an observation in mosaicing mode.

In order to cover the sky north of declination $30^{\circ}$, the sky was divided into four zones. Three zones are centered on declinations of $37^{\circ}, 50^{\circ}, 66^{\circ}$, with a different mosaic pattern used in each zone. The layout of these WENSS mosaics is displayed in Fig. 2. The polar cap, the fourth zone, will be discussed elsewhere.

An optimal trade-off between uniform sensitivity and efficiency would be obtained with a grid of fields with a regular spacing between fields that is equal to the halfpower width of the primary beam (HPBW). Technical considerations require a pattern of fields on a grid with an hour-angle separation in right ascension that is con-

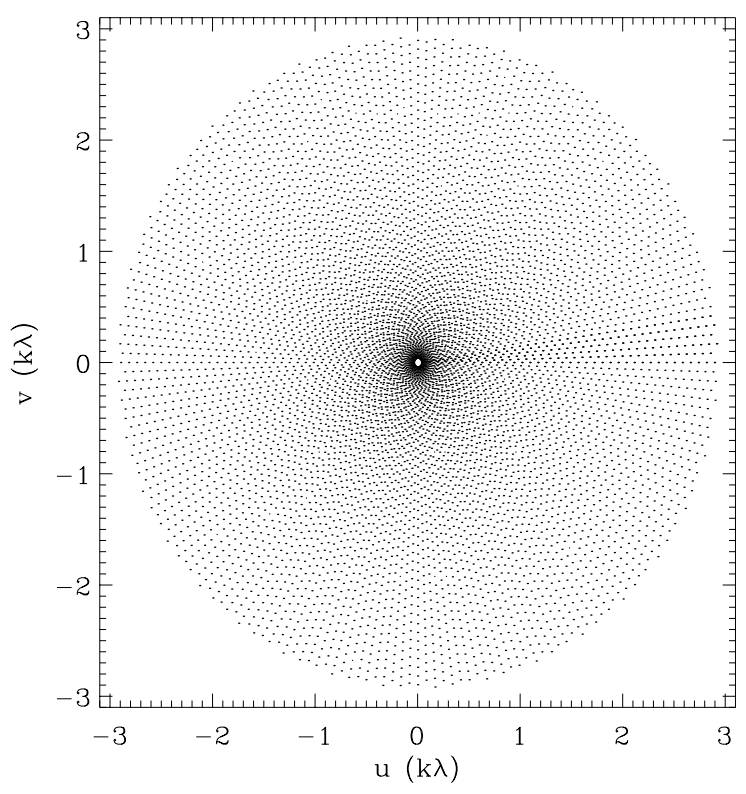

Fig. 1. $u, v$ coverage for a mosaic observation with 108 spokes, resulting from a combination of 18 spokes from 6 array configurations (12 meter increment). The scale is for a wavelength of $92 \mathrm{~cm}$ and for clarity the tracks are shown for an observation at $\delta=90^{\circ}$

stant. At low declination we therefore use grid patterns for the mosaics that are simple rectangular grids of $8 \times 10$ fields. At a declination of $66^{\circ}$ this would lead to a somewhat less efficient pattern since the actual spacing between adjacent points of constant RA decreases rather rapidly. This leads to the more complicated pattern of Fig. 3. At high declination the grid-separation in right ascension in this pattern is doubled. This is the pattern used by the mosaics that cover the mini-survey. The grid spacings in the three declination zones are listed in Table 2.

Table 2. Definition of the grids used in constructing the mosaics

\begin{tabular}{lccc}
\hline & & \multicolumn{2}{c}{ Grid separation } \\
Declination zone & Grid pattern & $\Delta \alpha\left(^{\mathrm{m}}\right)$ & $\Delta \delta\left(^{\circ}\right)$ \\
\hline $37^{\circ}$ & $8 \times 10$ & 6.60 & 1.33 \\
$50^{\circ}$ & $8 \times 10$ & 8.18 & 1.33 \\
$66^{\circ}$ & $6 \times 14$ & 12.74 & 1.33 \\
\hline
\end{tabular}

\subsection{Observations}

Observations for WENSS were carried out with the WSRT in a standard set-up. The mosaicing mode described previously prescribed the telescope configuration for the 


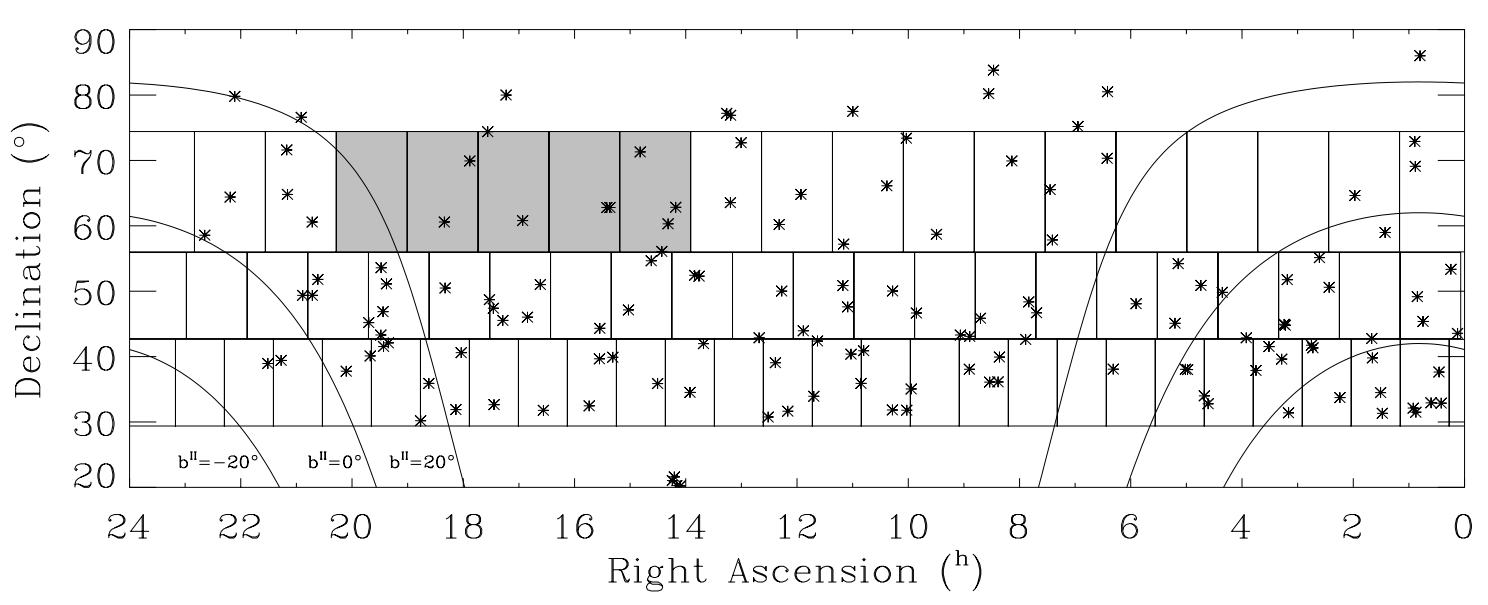

Fig. 2. Layout of the WENSS mosaics currently (March 1996) observed and processed. The five darker shaded mosaics comprise the mini-survey described later in this paper. The symbols mark strong (3C) radio sources. Lines of constant galactic latitude $\left(-20^{\circ}, 0^{\circ}\right.$, and $\left.+20^{\circ}\right)$ are indicated

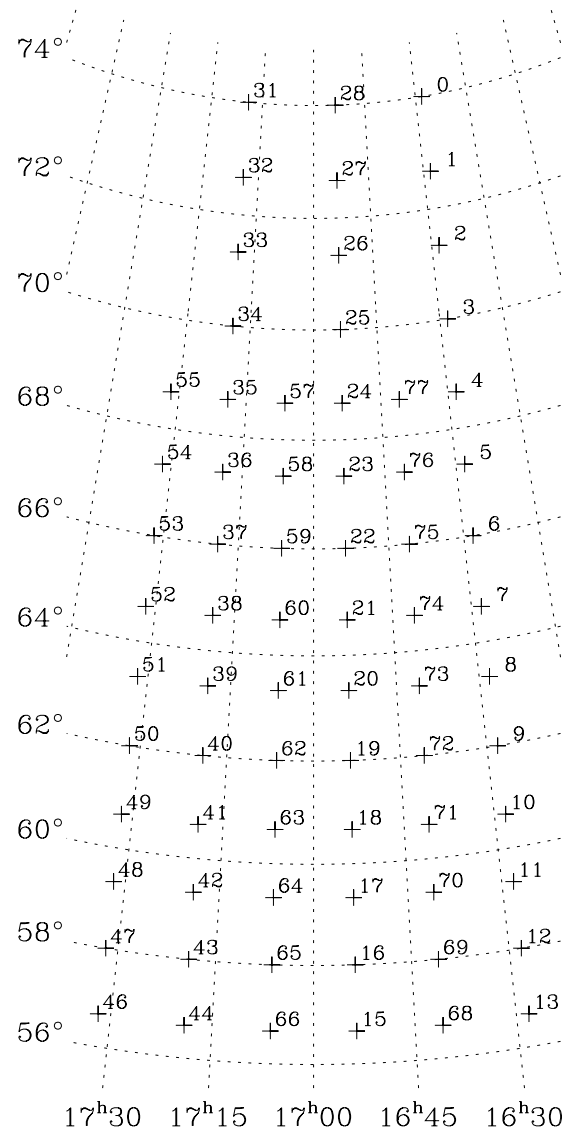

Fig. 3. The field pattern for the $\delta=66^{\circ}$ mosaics. In this case the pattern for mosaic WN66-255 is shown. The field are numbered according to the sequence of observation. Missing numbers refer to so-called "moving" pointings, inserted to bridge large field separations
WSRT. At $92 \mathrm{~cm}$ observations were carried out with the DXB backend, at a frequency of $325.125 \mathrm{MHz}$, with a total bandwidth of $5 \mathrm{MHz}$. In the winter of 19913 frequency channels were used. This was later changed to 7 frequency channels. The number of frequency channels has no notable influence on the quality of the maps.

\subsection{Reduction}

Mosaics were calibrated and reduced using the WSRT reduction package NEWSTAR (Netherlands East-West Synthesis Telescope Array Reduction). Initially, each field was calibrated and reduced separately in a way that is comparable to the calibration/reduction of standard 12 hour syntheses. This procedure started with the flagging of bad data and an absolute gain and phase calibration using one or more primary or secondary calibrators (3C 48 , 3C 147, 3C 286, and 3C 295). A "dirty" map was then made by a Fast Fourier transform (FFT) of the visibility data. The brightest sources from this map were selected and used to construct a first model. For this model the predicted visibilities were determined and subtracted from the visibility data. On the predicted visibilities we performed a phase-only self-calibration, which was then used to correct the residual visibility data. From the residual visibility data a new map was constructed and additional components for the model were extracted. The improved model was again used in a phase only self-calibration. This process was repeated a third and final time. (Wieringa 1991a). This process removes the time-dependent phase errors caused by the ionosphere. These phase errors are the dominant source of error deforming the sources. Only for fields with very strong (more than a few Jy) sources we also did a phase and gain self-calibration. (Wieringa 1991a). 
Since, at low frequency, the ionosphere introduces substantial absolute phase errors that are not corrected for in the self-calibration, each field can have an absolute position uncertainty of typically $5^{\prime \prime}$. The positions of each field within the mosaic were therefore corrected using a system of secondary position calibrators from the JVAS survey (Patnaik et al. 1992), combined with calibrators whose positions were obtained through pointed $21 \mathrm{~cm}$ WSRT observations. These latter calibrators were included to obtain a more uniform distribution of calibrator sources over the sky. Fields that did not contain position calibrators were tied into this system using additional strong sources that were present in adjacent overlapping fields.

In the final step of the reduction process, the individual fields were combined into a single mosaic. To do this the self-calibrated model-subtracted visibility data of all fields in a mosaic were Fourier-transformed onto the same reference grid. The residual maps were cleaned, using the CLEAN algorithm (Högbom 1974) and corrected for selfcal bias (Wieringa 1991a). The model and the clean-components were restored using a Gaussian restoring beam with a full-width at half maximum (FWHM) of $54^{\prime \prime} \times 54^{\prime \prime} \operatorname{cosec} \delta($ at $92 \mathrm{~cm})$. The maps of the individual fields were then added using a weight that is proportional to the sensitivity of each field at that position in the mosaic (i.e. inversely proportional to the square of the attenuation of the primary beam)

The reduction steps described here apply to the total intensity maps. The polarization maps require additional calibration steps that will be discussed elsewhere.

\subsection{Frame production}

From the mosaics, we made maps with a uniform sensitivity and a regular size. We call these maps frames. These frames were constructed in the same way as the maps for the mosaics, but can incorporate fields from different mosaics.

The $92 \mathrm{~cm}$ frames are $6 \times 6$ degree in size, and positioned on a regular $5 \times 5$ degree grid over the sky. This grid coincides with the position grid of the new Palomar Observatory Sky Survey (POSS) plates. All frames have a standard $1024 \times 1024$ pixel format, and use the WSRTspecific north-polar cap (NPC) projection. This projection is defined by the following relation between the pixel $(x, y)$ and celestial coordinates $(\alpha, \delta)$ :

$$
\begin{aligned}
& \left(x-x_{0}\right) \Delta x=-\cos \delta \sin \left(\alpha-\alpha_{0}\right) \\
& \left(y-y_{0}\right) \Delta y=\frac{\cos \delta_{0}-\cos \delta \cos \left(\alpha-\alpha_{0}\right)}{\sin \delta_{0}}
\end{aligned}
$$

with the reference pixel $\left(x_{0}, y_{0}\right)=(512,512)$, and the pixel size $\Delta x=\Delta y \approx 21.1^{\prime \prime} \mathrm{pix}^{-1}$, the same for all 92 $\mathrm{cm}$ frames. The reference position $\alpha_{0}, \delta_{0}$ is given in B1950 coordinates.

\subsection{Source extraction}

A procedure to extract a list of discrete radio sources from a frame was written in IDL, the Interactive Data Language.

The procedure starts with obtaining the noise level $\sigma_{\text {rms }}\left(x_{i}, y_{i}\right)$ at each point in the map, by interpolating the rms-noises for a regular grid of fields (size: $96 \times 96$ pixels) within the map. A $\chi^{2}$ minimization fit of a Gaussian to the intensity distribution establishes an rms-noise level for each of these fields. We then use the following, recursive, definition of a source, based on the appearance of a distinct "island" of detected brightness in the map:

Given a pixel of intensity $I\left(x_{i}, y_{i}\right)>4 \sigma_{\mathrm{rms}}\left(x_{i}, y_{i}\right)$, the set of all the pixels $I\left(x_{j}, y_{j}\right)>2.5 \sigma_{\mathrm{rms}}\left(x_{j}, y_{j}\right)$ that are adjacent to either this pixel, or another pixel within this set, constitutes an island. This island we call a source. To obtain a more realistic estimate of the integrated flux, taking account of the noise, all pixels directly adjacent to this set are added to the island.

A local maximum is defined as a pixel whose intensity $I\left(x_{i}, y_{i}\right)>4 \sigma_{\mathrm{rms}}$ is larger than all eight surrounding pixels. Based on the number of local maxima within the island, we distinguish: single-component ("S") sources, with 1 local maximum, multiple component ("M") sources, with 2-4 local maxima, and extended ("E") sources, with more than 4 local maxima.

The relevant source parameters are: position $(x, y)$, peak and integrated flux density $(S, S I)$, the size of the major and minor axes (FWHM, $\left.b_{\mathrm{Max}}, b_{\min }\right)$, and the position angle $\left(\Theta_{\mathrm{PA}}\right)$. For each source, these parameters are first computed from the brightness distribution $I\left(x_{i}, y_{i}\right)$ using weighted moment analysis, with the peak flux $S_{\mathrm{m}}=\max \left(I\left(x_{i}, y_{i}\right)\right)$, and the integrated flux $S I_{\mathrm{m}}=$ $\sum_{i} I\left(x_{i}, y_{i}\right) /$ beam, with the beam $=\pi B_{\mathrm{M}} B_{\mathrm{m}} / 4 \ln 2$, and $B_{\mathrm{M}}$ and $B_{\mathrm{m}}$ the FWHM of the major and minor axis of the restoring beam. The remaining parameters are computed from the weighted first and second order moments:

$m_{k l}=\frac{\sum_{i} x_{i}^{k} y_{i}^{l} I\left(x_{i}, y_{i}\right)}{\sum_{i} I\left(x_{i}, y_{i}\right)}$,

with: $x=m_{10}, y=m_{01}, \tan 2 \Theta_{\mathrm{PA}}=2 m_{11} /\left(m_{20}-m_{02}\right)$, and the eccentricity $e=\sqrt{\left(m_{20}-m_{02}\right)^{2}+4 m_{11}^{2}} /\left(m_{20}+\right.$ $\left.m_{02}\right) . b_{\text {Maj }}$ and $b_{\min }$ are solved from the eccentricity and the ratio $S I / S=b_{\mathrm{M}} b_{\mathrm{m}} / B_{\mathrm{M}} B_{\mathrm{m}}$.

An attempt is made to fit an "S" or "M" source with a model consisting of a number of elliptical Gaussians equal to the number of local maxima. The Gaussians are parameterized by:

$I(x, y)=p_{1} \mathrm{e}^{\frac{-\ln 2}{1-p_{6}^{2}}\left\{\left(\frac{x-p_{2}}{p_{4}}\right)^{2}+\left(\frac{y-p_{3}}{p_{5}}\right)^{2}-2 p_{6} \frac{\left(x-p_{2}\right)(y-p 3)}{p_{4} p_{5}}\right\}}$.

The fitting-algorithm is based on the LevenbergMarquardt algorithm from Numerical Recipes (Press et al. 1992). The parameters $p_{1}-p_{6}$ from these fits are converted 
Table 3. The $92 \mathrm{~cm}$ mosaics included in the mini-survey. Listed are the nominal mosaic center and the epochs of observation for the different telescope configurations, defined by the distance between telescopes " 9 " and "A"

\begin{tabular}{lcccccccc}
\hline Mosaic & \multicolumn{2}{c}{ Mosaic center (B1950) } & \multicolumn{5}{c}{ Epochs of observation (yymmdd) } \\
& Right Ascension & Declination & $36 \mathrm{~m}$ & $48 \mathrm{~m}$ & $60 \mathrm{~m}$ & $72 \mathrm{~m}$ & $84 \mathrm{~m}$ & $96 \mathrm{~m}$ \\
\hline WN66_217 & $14^{\mathrm{h}} 26^{\mathrm{m}}$ & $66^{\circ} 00^{\prime}$ & 930201 & 930131 & 940416 & 930102 & 930128 & 930126 \\
WN66_236 & $15^{\mathrm{h}} 43^{\mathrm{m}}$ & $66^{\circ} 00^{\prime}$ & 920112 & 920214 & 930109 & 911201 & 911215 & 911222 \\
WN66_255 & $16^{\mathrm{h}} 59^{\mathrm{m}}$ & $66^{\circ} 00^{\prime}$ & 910216 & 910223 & 910311 & 910114 & 910205 & 910208 \\
WN66_274 & $18^{\mathrm{h}} 16^{\mathrm{m}}$ & $66^{\circ} 00^{\prime}$ & 910217 & 910224 & 910302 & 910120 & 910202 & 910209 \\
WN66_293 & $19^{\mathrm{h}} 32^{\mathrm{m}}$ & $66^{\circ} 00^{\prime}$ & 910218 & 910225 & 910303 & 910329 & 910201 & 910210 \\
\hline
\end{tabular}

Table 4. The high-resolution $92 \mathrm{~cm}$ frames included in the mini-survey

\begin{tabular}{lcc}
\hline Frame & \multicolumn{2}{c}{ Map Center $($ B1950) } \\
& RA & Dec \\
\hline WNH60_218 & $14^{\mathrm{h}} 34^{\mathrm{m}} 00^{\mathrm{s}}$ & $60^{\circ} 00^{\prime}$ \\
WNH60_228 & $15^{\mathrm{h}} 12^{\mathrm{m}} 00^{\mathrm{s}}$ & $60^{\circ} 00^{\prime}$ \\
WNH60_237 & $15^{\mathrm{h}} 50^{\mathrm{m}} 00^{\mathrm{s}}$ & $60^{\circ} 00^{\prime}$ \\
WNH60_247 & $16^{\mathrm{h}} 28^{\mathrm{m}} 00^{\mathrm{s}}$ & $60^{\circ} 00^{\prime}$ \\
WNH60_256 & $17^{\mathrm{h}} 06^{\mathrm{m}} 00^{\mathrm{s}}$ & $60^{\circ} 00^{\prime}$ \\
WNH60_266 & $17^{\mathrm{h}} 44^{\mathrm{m}} 00^{\mathrm{s}}$ & $60^{\circ} 00^{\prime}$ \\
WNH60_275 & $18^{\mathrm{h}} 22^{\mathrm{m}} 00^{\mathrm{s}}$ & $60^{\circ} 00^{\prime}$ \\
WNH60_285 & $19^{\mathrm{h}} 00^{\mathrm{m}} 00^{\mathrm{s}}$ & $60^{\circ} 00^{\prime}$ \\
WNH60_294 & $19^{\mathrm{h}} 38^{\mathrm{m}} 00^{\mathrm{s}}$ & $60^{\circ} 00^{\prime}$ \\
WNH65_220 & $14^{\mathrm{h}} 40^{\mathrm{m}} 00^{\mathrm{s}}$ & $65^{\circ} 00^{\prime}$ \\
WNH65_231 & $15^{\mathrm{h}} 24^{\mathrm{m}} 00^{\mathrm{s}}$ & $65^{\circ} 00^{\prime}$ \\
WNH65_242 & $16^{\mathrm{h}} 08^{\mathrm{m}} 00^{\mathrm{s}}$ & $65^{\circ} 00^{\prime}$ \\
WNH65_253 & $16^{\mathrm{h}} 52^{\mathrm{m}} 00^{\mathrm{s}}$ & $65^{\circ} 00^{\prime}$ \\
WNH65_264 & $17^{\mathrm{h}} 36^{\mathrm{m}} 00^{\mathrm{s}}$ & $65^{\circ} 00^{\prime}$ \\
WNH65_275 & $18^{\mathrm{h}} 20^{\mathrm{m}} 00^{\mathrm{s}}$ & $65^{\circ} 00^{\prime}$ \\
WNH65_286 & $19^{\mathrm{h}} 04^{\mathrm{m}} 00^{\mathrm{s}}$ & $65^{\circ} 00^{\prime}$ \\
WNH65_297 & $19^{\mathrm{h}} 48^{\mathrm{m}} 00^{\mathrm{s}}$ & $65^{\circ} 00^{\prime}$ \\
WNH70_221 & $14^{\mathrm{h}} 43^{\mathrm{m}} 00^{\mathrm{s}}$ & $70^{\circ} 00^{\prime}$ \\
WNH70_234 & $15^{\mathrm{h}} 36^{\mathrm{m}} 00^{\mathrm{s}}$ & $70^{\circ} 00^{\prime}$ \\
WNH70_247 & $16^{\mathrm{h}} 28^{\mathrm{m}} 00^{\mathrm{s}}$ & $70^{\circ} 00^{\prime}$ \\
WNH70_260 & $17^{\mathrm{h}} 20^{\mathrm{m}} 00^{\mathrm{s}}$ & $70^{\circ} 00^{\prime}$ \\
WNH70_273 & $18^{\mathrm{h}} 12^{\mathrm{m}} 00^{\mathrm{s}}$ & $70^{\circ} 00^{\prime}$ \\
WNH70_286 & $19^{\mathrm{h}} 04^{\mathrm{m}} 00^{\mathrm{s}}$ & $70^{\circ} 00^{\prime}$ \\
WNH70_299 & $19^{\mathrm{h}} 56^{\mathrm{m}} 00^{\mathrm{s}}$ & $70^{\circ} 00^{\prime}$ \\
\hline & & \\
& &
\end{tabular}

to position, flux densities, major and minor axis and position angle and used to describe the source. If the algorithm fails to properly fit a source are the values from moment analysis used to parameterize the source. The values from moment analysis are also used for "E" sources. For "M" sources as a whole the position and morphology are established through moment analysis, while the peak flux density is the maximum of the peak flux densities of the components and the integrated flux density is the sum of the integrated flux densities of the components.

We find that the estimates of the flux densities and the source morphology are affected by biases at low signal-to- noise ratios. We therefore apply empirical corrections to the flux-density estimates. These corrections are discussed in Sect. 3.4.

\section{The mini-survey}

The WENSS project initially concentrated on a relatively small area of sky roughly centered on the North Ecliptic Pole. This region was chosen to coincide with the NEPVLA survey at $1.5 \mathrm{GHz}$ (Kollgaard et al. 1995), the deep 7C North Ecliptic Cap survey (Lacy et al. 1995; Visser et al. 1995), and the deepest part of the ROSAT All Sky survey (Böhringer et al. 1991; Bower et al. 1996) and IRAS survey (Hacking \& Houck 1987).

Our original intention was to base our complete analysis of errors and reliability on the mini-survey region. However, data from various other parts of the survey have been included in this analysis. Nevertheless the minisurvey constitutes the most thoroughly analyzed part of WENSS to date. Furthermore, data from the mini-survey has already been used extensively for various followup projects, including a search for gravitational lenses (CLASS, Myers et al. 1995; Snellen et al. 1995), and investigations of samples of faint Gigaherz peaked spectrum sources and faint ultra steep spectrum sources.

\subsection{Observations}

Frames were constructed from the five mosaics listed in Table 3 . The mosaics are labeled by the declination and approximate right-ascension of their center. Four of mosaics were observed at the start of the WENSS project in the spring of 1991. Mosaic WN66 - 217 was later included in the mini-survey to improve the overlap with the 7C survey (Visser et al. 1995). Figure 2 shows the layout of these mosaics within the the survey.

A list of the 24 high resolution $92 \mathrm{~cm}$ frames included in the mini-survey is presented in Table 4. The frames are labeled by the declination and approximate rightascension of their center. Figure 4 shows the layout of these fields.

The theoretical noise level for WENSS is approximately $2 \mathrm{mJy}$ beam $^{-1}$. Sidelobe confusion increases this 


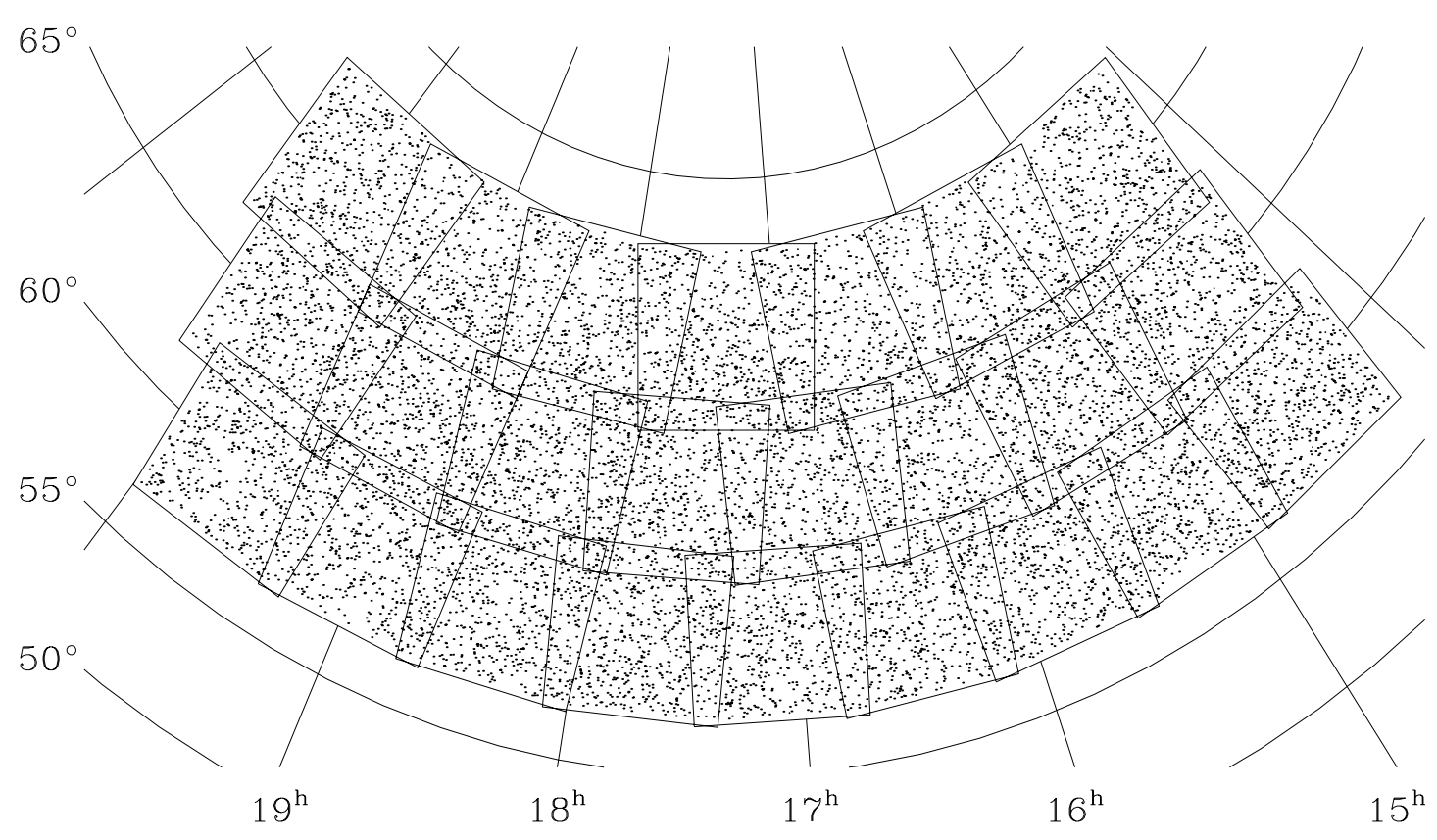

Fig. 4. The layout of the frames included in the mini-survey. The dots mark the individual sources assembled in the source list

to about $3-4$ mJy beam ${ }^{-1}$. Figure 5 shows the distribution of the local noise-level within the mini-survey. We estimate the noise level to be determined with an accuracy better than 0.2 mJy beam ${ }^{-1}$. The noise level is on average $3.9 \mathrm{mJy}^{\text {beam }}{ }^{-1}$, and varies between 2.7 and $6.7 \mathrm{mJy}$ beam $^{-1}$. Over $95 \%(99 \%)$ of the area the noise level is smaller than $4.9(5.5) \mathrm{mJy}_{\text {beam }}{ }^{-1}$. The variation is therefore more than an order of magnitude larger than the error in the estimate of the noise, and is caused by systematic effects. To illustrate this, the map in Fig. 6 shows the spatial structure of the variation. Note that the variation in the noise level shows a correlation length of many degrees, and that the noise level varies smoothly between frames. From the location of the brightest $4 \mathrm{C}$ sources in this region, it can be clearly seen that some regions of enhanced local noise are associated with strong radio sources. This is due to sidelobe confusion.

Figure 6 also indicates a variation in noise level as a function of declination. Going from low to intermediate declination the noise-level decreases as the effective spacing between individual fields, positioned at points of constant right ascension, decreases. At higher declination the spacing in right ascension between field is raised to increase the efficiency of the mosaicing observations (see Fig. 3). This leads to an increase in the noise level at higher declinations.

Finally, interference leads to a non-uniform quality of the data for different fields, resulting in noise variations over the survey area.

\subsection{The catalogue}

A source list for each frame was compiled using the sourceextraction software described previously. The resultant lists were then combined into a final source list. In the case of multiple entries from a source appearing in more than one frame, only the entry with the best signal-tonoise ratio was included. Table 5 shows a sample of the source list. The complete catalogue of 11299 sources included in the twenty-four frames of the mini-survey can be found in Table 6. For the 477 multiple component sources, the 994 components are also included. This table is only available in electronic form as an ASKII-table at the CDS via anonymous ftp to cdsarc.u-strasbg.fr (130.79.128.5) or via http://cdsweb.u-strasbg.fr/Abstract.html. For each source we list:

Name This follows the IAU convention of naming sources according to their position $(\mathrm{Bhhmm}+d d m \mathrm{~m})$. As a prefix to the name we use "WN", which stands for WENSS Ninety $\mathrm{cm}$. Each multiple component source has one entry for the source as a whole, and one entry for each of its components, designated "A", "B", etc.

Position Right ascension and declination are given in B1950 coordinates.

Type Sources are distinguished according to the number of Gaussian components used in an attempt to model them. "S" or single-component sources have been modeled with one Gaussian. "M" or multi-component sources were fit with up to four Gaussians, each of them listed as a type "C" source. No attempt to model the "E" (extended) sources was made. 

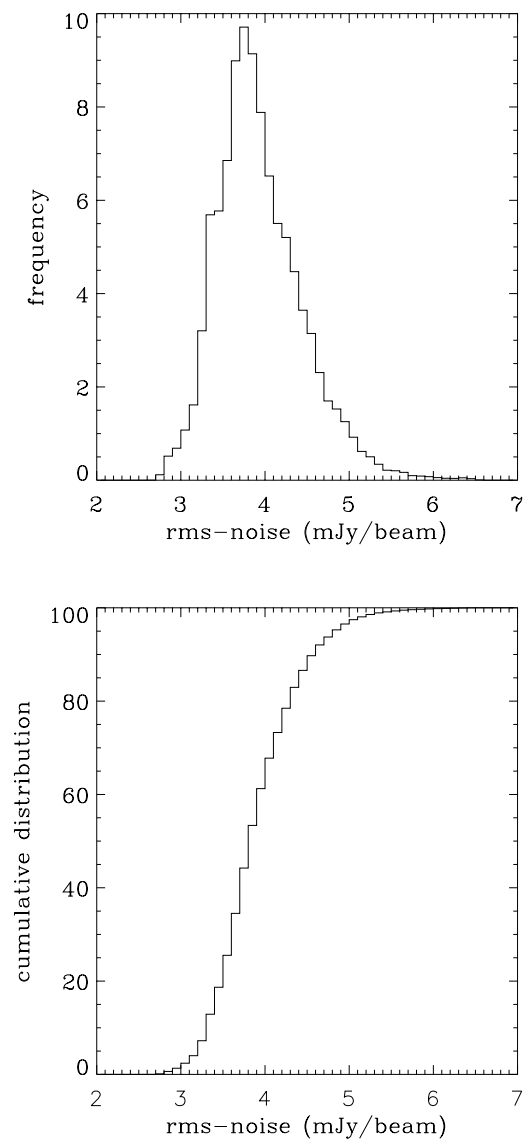

Fig. 5. The distribution (top) and cumulative distribution (bottom) of rms-noise levels in the mini-survey

Flag If, for any reason, the source-finding algorithm failed to fit a source, then the source parameters were obtained through moment-analysis. These sources are marked with an asterisk "*".

Flux Peak $\left(S\right.$, mJy beam $\left.^{-1}\right)$ and integrated $(S I$, mJy) flux-densities are listed.

Morphology The source morphology is characterized by the major and minor axes, $b_{\mathrm{Maj}}, b_{\min }$, and the position angle $\Theta_{\mathrm{PA}} . b_{\mathrm{Maj}}$ and $b_{\mathrm{min}}$ are in arcseconds, $\Theta_{\mathrm{PA}}$ is measured in degrees from north through east. Values are only listed for resolved sources/components (see below).

Noise The local rms-noise level in mJy beam ${ }^{-1}$.

Frame The frame from which the source was obtained.

Single-component sources are divided into categories of resolved and unresolved sources. The ratio of integrated to peak flux $S I / S$ was used to distinguish between these categories. From Monte-Carlo simulations we found the flux ratio as a function of signal-to-noise ratio below which $95 \%$ of the unresolved sources are located. We consider all sources with a flux ratio exceeding this $95 \%$ limit to be possibly resolved. A numerical expression for this ratio is given below (Eq. 10). Note that the values for $b_{\mathrm{Maj}}, b_{\mathrm{min}}$ and $\Theta_{\mathrm{PA}}$ have not been deconvolved to correct for the beam.

The total number of sources in each category is listed in Table 7. This table also shows the number of sources in each category for which the source finding algorithm failed to find a good fit. The percentage is especially high $(9 \%)$ for resolved single component sources. If the source finding algorithm failed to find a good fit for an "M" source, then it was not able to establish parameters of individual components, other than the position and peak flux density of the local maximum.

\subsection{Monte-Carlo simulations}

To assess the reliability of the source parameters obtained in the analysis of the maps, we used Monte-Carlo simulations. In these simulations Gaussian intensity distributions were added to empty regions in several frames. The major- and minor axes of these distributions were those of the restoring beam. The maximum intensity was varied during the simulation. These sources were then analyzed using the standard source finding algorithm. In this way the distributions of the various parameters as a function of signal-to-noise ratio was established. These distributions were used to investigate biases in the parameters and estimate the errors for the parameters.

\subsection{Flux correction}

From the Monte-Carlo simulations, it was found that the estimates of the flux densities are systematically affected by sampling and noise.

In general, the position on the sky of the pixel with the maximum detected brightness does not coincide with the actual location of the maximum of the source. The pixel with maximum detected brightness in an island measures, in the absence of noise, the flux density at the pixel nearest to the peak. On average we find that the pixel with the maximum detected brightness underestimates the peak flux density by $6 \%$. We therefore adopt the following overall correction to the peak flux density $\left(S_{\mathrm{m}}\right)$ as measured from moment analysis,

$S_{\mathrm{m}}^{\prime}=1.06 S_{\mathrm{m}}$.

Since the edge of an island is defined by the $2.5 \sigma_{\mathrm{rms}}$ contour, the area over which the integrated flux density is measured is a function of the signal-to-noise ratio. This effect will lead to an underestimate of the integrated flux densities measured from moment analysis.

Another bias is introduced by incorporating positive noise peaks (including undetected weak sources) within an island, adding to the integrated flux density, while excluding negative noise peaks from the island, especially when they occur at the fringes of the island. This effect will partly reduce the underestimate of the integrated flux 
Table 5. A sample from the catalogue for the mini-survey area. The complete catalogue (Table 6) is only available in electronic form. For each source we list: name, position (B1950), source type, a flag, peak and itegrated flux densities, local noise level, morphological parameters, and the frame from which the source was taken

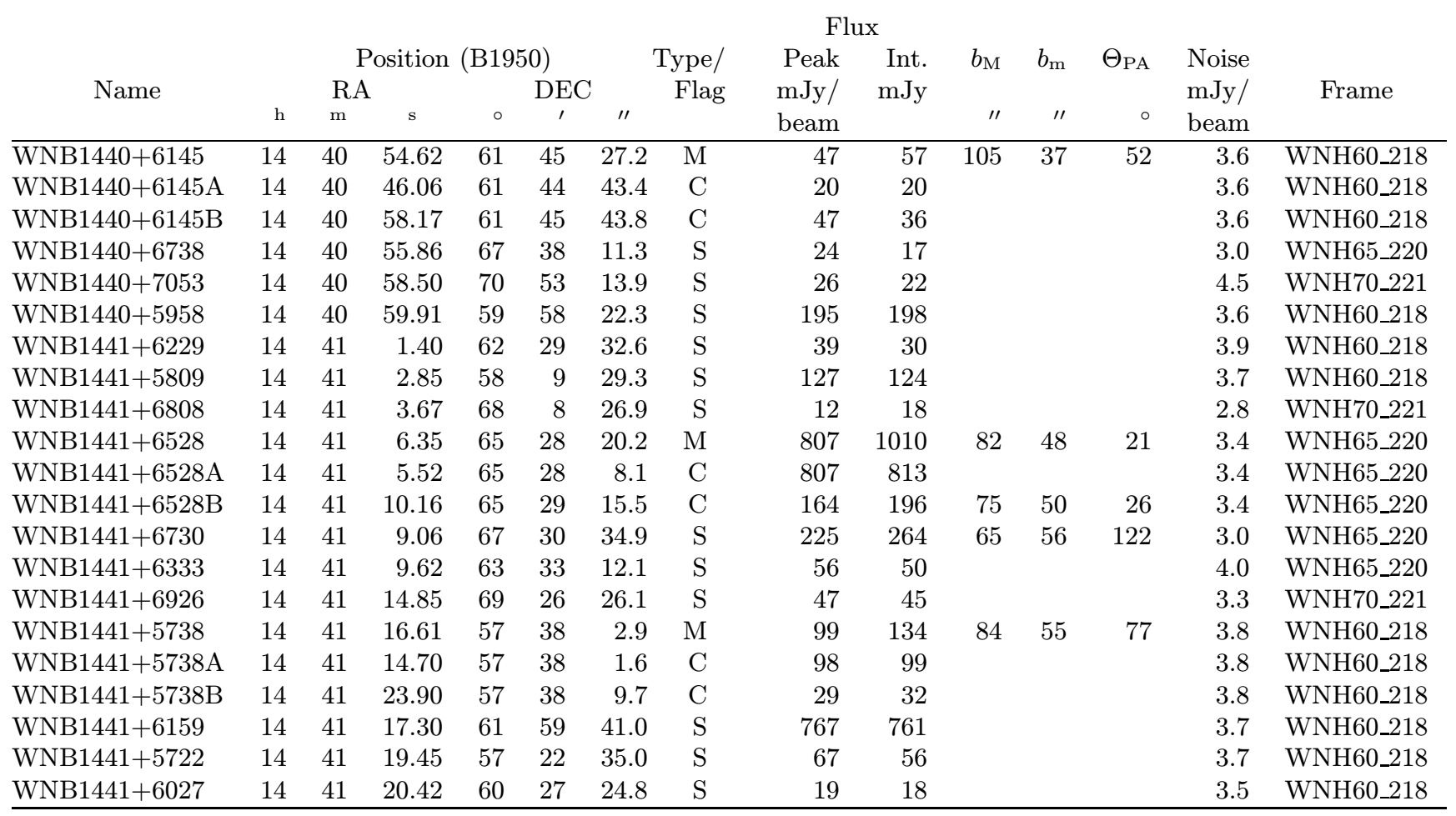

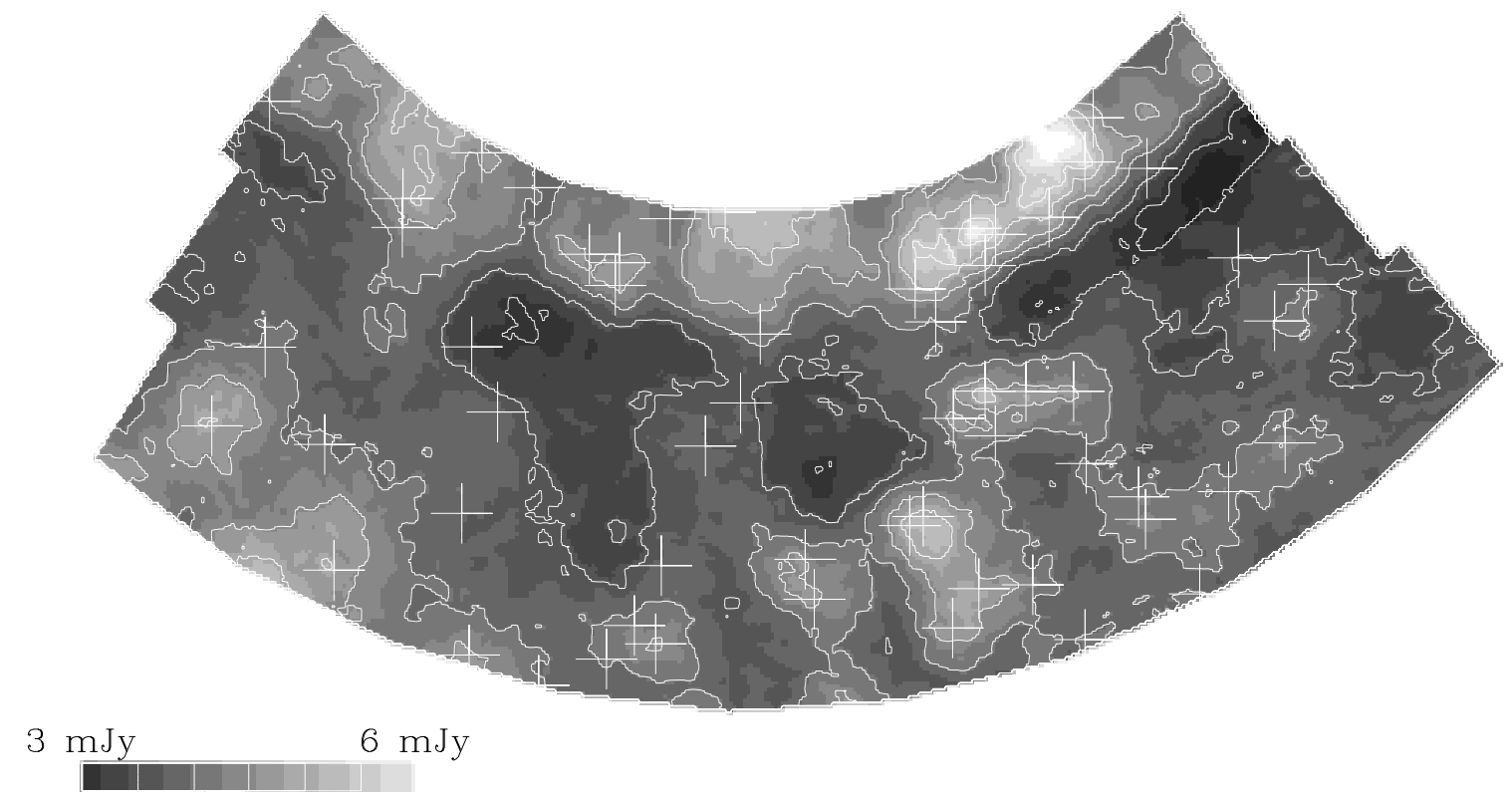

Fig. 6. Variation of the noise level over the area of the mini-survey. Contour levels are at noise-levels of $3,3.5,4,4.5,5$ and $6 \mathrm{mJy}^{\text {beam }}{ }^{-1}$. Crosses mark the position of $4 \mathrm{C}$ sources with $S_{178 \mathrm{MHz}}>3 \mathrm{Jy}$ 
Table 7. Number of sources within each category. For each category the number of sources with problems is also listed. For these sources parameters were derived from moment analysis rather than elliptical Gaussian distributions. Numbers within parentheses are percentages of the total number of sources (11 299)

\begin{tabular}{lrrrr}
\hline Category & & \multicolumn{3}{c}{ with flag “*” } \\
\hline S sources (unresolved) & 8961 & $(79.3 \%)$ & 110 & $(1.0 \%)$ \\
S sources (resolved) & 1858 & $(16.4 \%)$ & 163 & $(1.4 \%)$ \\
M sources (2 comp's) & 443 & $(4.0 \%)$ & 11 & $(0.1 \%)$ \\
M sources (3 comp's) & 28 & $(0.2 \%)$ & 0 & \\
M sources (4 comp's) & 6 & $(<0.1 \%)$ & 0 & \\
E sources & $\mathrm{a}$ & & 3 & $(<0.1 \%)$ \\
C sources (unresolved) & 713 & & 22 & \\
C sources (resolved) & 259 & & - & \\
\hline
\end{tabular}

Notes: a) No attempt to fit "E"-sources was made.

densities measured from moment analysis. We find that for the fit-routine this results in a higher estimate of the peak flux, a higher estimate of the integrated flux, and a lower estimate of the source extension.

All biases discussed here are small $(<5 \%)$, and only occur at small signal-to-noise ratios $\left(S / \sigma_{\text {rms }}<10\right)$, with biases only of the order of $2 \%$ at a signal-to-noise ratio of 8 . Note that the random errors in the flux density measurements in this regime range from approximately $15 \%$ to $25 \%$ (see below). Nevertheless, we introduce empirical corrections to the integrated $\left(S I_{\mathrm{m}}\right)$ flux density from moment analysis, and the peak $\left(S_{\mathrm{f}}\right)$ and integrated $\left(S I_{\mathrm{f}}\right)$ flux densities from fitting. These corrections are only applied to sources for which the signal-to-noise ratio of the peak pixel $S_{\mathrm{m}}^{\prime} / \sigma_{\mathrm{rms}}<10$. The correction factors are smooth functions of the logarithm of the signal-to-noise ratio, ranging from 1 at $\log \left(S_{\mathrm{m}}^{\prime} / \sigma_{\mathrm{rms}}\right)=1$ to approximately 1.06 at $\log \left(S_{\mathrm{m}}^{\prime} / \sigma_{\mathrm{rms}}\right)=0.7$

$$
\begin{array}{ccc}
S I_{\mathrm{m}}^{\prime}= & S I_{\mathrm{m}} /\left(0.8+0.2 \log S_{\mathrm{m}}^{\prime} / \sigma_{\mathrm{rms}}\right) \\
S I_{\mathrm{f}}^{\prime}= & S I_{\mathrm{f}} /\left(1.2-0.2 \log S_{\mathrm{m}}^{\prime} / \sigma_{\mathrm{rms}}\right) \\
S_{\mathrm{f}}^{\prime}= & S_{\mathrm{f}} /\left(1.6-1.2 \log S_{\mathrm{m}}^{\prime} / \sigma_{\mathrm{rms}}+0.6 \log ^{2} S_{\mathrm{m}}^{\prime} / \sigma_{\mathrm{rms}}\right) \\
& \text { with: } \log S_{\mathrm{m}}^{\prime} / \sigma_{\mathrm{rms}}<1 .
\end{array}
$$

The estimates of the major and minor axis $\left(b_{\mathrm{Maj}}, b_{\mathrm{min}}\right)$ are adjusted to conserve the relation $S I=$ $S b_{\mathrm{M}} b_{\mathrm{m}} /\left(B_{\mathrm{M}} B_{\mathrm{m}}\right)$, with $B_{\mathrm{M}}, B_{\mathrm{m}}$ the major and minor axes of the beam. Thus:

$$
\begin{aligned}
& b_{\mathrm{Maj}}^{\prime}=b_{\mathrm{Maj}}\left(\frac{S I_{\mathrm{f}}^{\prime}}{S I_{\mathrm{f}}} \frac{S_{\mathrm{f}}}{S_{\mathrm{f}}^{\prime}}\right)^{\frac{1}{2}} \\
& b_{\min }^{\prime}=b_{\min }\left(\frac{S I_{\mathrm{f}}^{\prime}}{S I_{\mathrm{f}}} \frac{S_{\mathrm{f}}}{S_{\mathrm{f}}^{\prime}}\right)^{\frac{1}{2}} .
\end{aligned}
$$

Note that although we search for sources down to a level of $4 \sigma_{\mathrm{rms}}$, in our source lists we only include sources for which the corrected peak flux $S_{\mathrm{m}}^{\prime}>5 \sigma_{\mathrm{rms}}$. This cut avoids corrections for systematic effects at very low signalto-noise ratios that could be as high as $20 \%$, but are very difficult to establish exactly.

\subsection{Error estimates}

In this section we discuss the errors in the estimates for the position, the peak and integrated flux densities, and the major and minor axes for each source.

It is important to note that the angle over which the rms noise is correlated is comparable to the beamsize. Analytical derivations of the errors in the estimates for the source parameters, based on the assumption of uncorrelated noise, are therefore not valid (See for example: Condon 1996). Empirical expressions, obtained from the Monte-Carlo simulations discussed previously, are therefore used to provide an estimate of the errors for the various parameters. These errors incorporate the random errors introduced by the rms noise as well as the systematic errors introduced by the fit procedures.

We find that all errors can be approximated by a quadratic sum of a systematic part and a signal-to-noise ratio dependent part, i.e:

$\sigma=\left\{C_{1}^{2}+C_{2}^{2}\left(\frac{\sigma_{\mathrm{rms}}}{S}\right)^{2}\right\}^{\frac{1}{2}}$.

The position and flux density measurements are compared with independent data to assess the quality of the data and to search for any systematic errors not included in the Monte-Carlo analysis.

Position Positional errors can be computed from:

$\sigma_{\alpha, \delta}=\left\{(1.5)^{2}+\left(\frac{\sigma_{\mathrm{rms}} \theta_{\alpha, \delta}}{1.3 S}\right)^{2}\right\}^{\frac{1}{2}}$,

where $\sigma_{\text {rms }}$ is the local rms-noise, $S$ the peak flux, and $\theta_{\alpha, \delta}$ is the source size. For strong sources this amounts to a position error of 1.5 arcsec in both right ascension and declination. The factor 1.3 in the above equation differs from the usual factor of 2 (Kaper et al. 1966). This is due to the correlation length of the noise. The factor was established using the Monte-Carlo simulations.

We have checked the positional accuracy, using two different samples.

A bright sample of sources was selected from the minisurvey, under the assumption that flat-spectrum sources are predominantly quasars of which a substantial fraction shows an optical counterpart on the Palomar Optical Sky Survey plates. This sample comprises 77 sources with $S>150 \mathrm{mJy}$, a spectral index $\alpha_{4850}^{327}>-0.5\left(S \propto \nu^{\alpha}\right.$, $S_{4850}$ from Gregory et al. 1996), and an optical candidate identification (ID) within 10". These IDs were obtained from the Cambridge APM catalogue (Irwin et al. 1994). Figure $7 \mathrm{a}$ shows, as diamonds, the position of candidate 

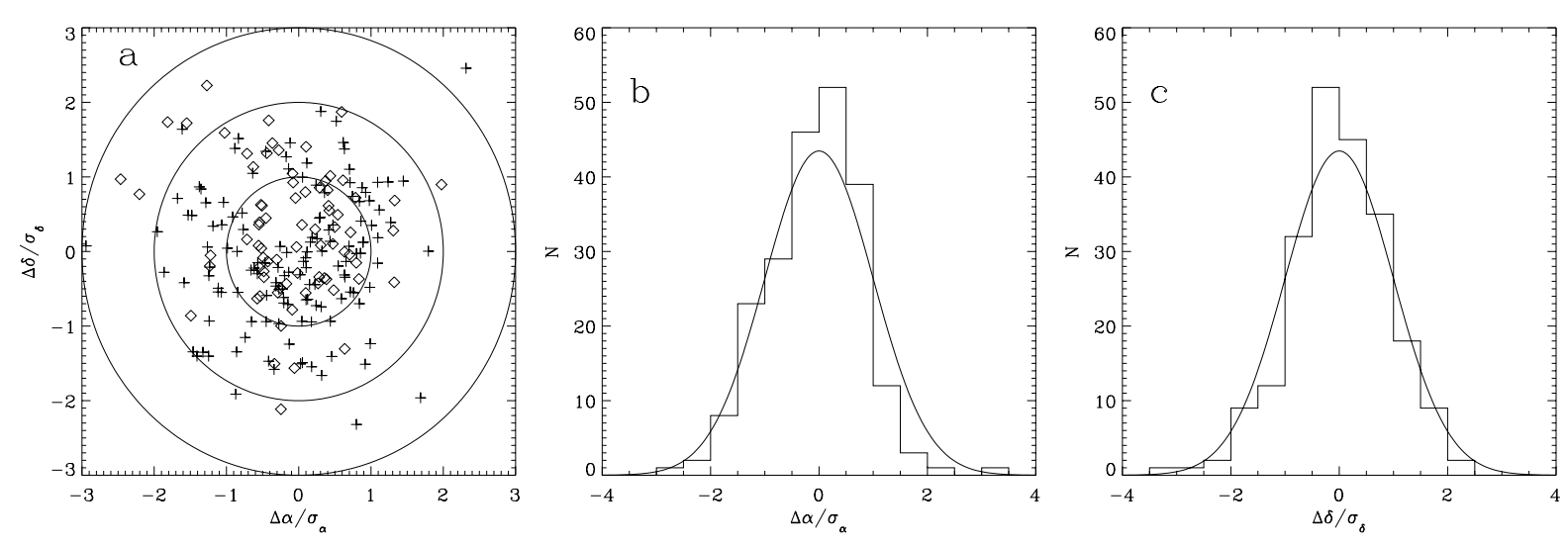

Fig. 7. a) Normalized position difference for candidate optical IDs of flat spectrum radio sources (diamonds) and for VLA positions (crosses). The concentric circles mark the 1,2, and $3 \sigma$ position differences respectively. b-c) The distribution of normalized position differences in right ascension and declination. Overlayed are the expected Gaussian distributions

IDs with respect to the radio position, normalized by the estimate of the errors in $\alpha$ and $\delta$. The errors were computed by adding the errors from Eq. (8) in quadrature to a positional error of $1^{\prime \prime}$ for the optical ID.

A faint sample was obtained from preliminary results of the CLASS gravitational lens survey (Myers et al. 1995). As part of this survey a large number of faint $(S<200 \mathrm{mJy})$, flat-spectrum $(\alpha>-0.5)$ WENSS sources was mapped using the VLA at $8.5 \mathrm{GHz}$ in A-array, to search for a characteristic gravitational lens morphology. Accurate $\left(\sigma<1^{\prime \prime}\right)$ positions for these sources were obtained as a by-product. Figure 7a shows, as crosses, the VLA positions, with respect to the WENSS positions, normalized by the errors in right ascension and declination.

Figures $7 \mathrm{~b}, \mathrm{c}$ show the combined distribution of position differences of both samples. These figures indicate that the error estimates are probably conservative in the sense that they overestimate the variance in the position difference. However, this overestimate allows for some possible systematic offsets at the $0.5^{\prime \prime}$ level, as indicated by the skew distribution in right ascension.

Flux densities The relative errors in the flux densities, can be computed from:

$\frac{\sigma_{S}}{S}=\left\{C_{1}^{2}+C_{2}^{2}\left(\frac{\sigma_{\mathrm{rms}}}{S}\right)^{2}\right\}^{\frac{1}{2}}$

with $S / \sigma_{\text {rms }}$ the signal-to-noise ratio. The values for the constants depend on the parameter being measured, and can be read from the following Table 8 .

The constant $C_{2}$ was estimated from Monte-Carlo simulations for unresolved sources. A conservative estimate for $C_{1}$ includes a $3 \%$ upper limit to the accuracy of the reduction process and source-finding algorithm and $\mathrm{a}<2 \%$ variation in the flux calibration for different mosaics. For the peak flux we add $5 \%$ to the error for the estimate
Table 8. Numerical values for the constants $C_{1}$ and $C_{2}$ in Eq. (9), determining the errors in the flux density estimates

\begin{tabular}{llll}
\hline Flux density (method) & $(S)$ & $C_{1}$ & $C_{2}$ \\
\hline Peak (moment) & $\left(S_{\mathrm{m}}\right)$ & 0.06 & 1.0 \\
Integr. (moment) & $\left(S I_{\mathrm{m}}\right)$ & 0.04 & 1.7 \\
Peak (fit) & $\left(S_{\mathrm{f}}\right)$ & 0.04 & 1.3 \\
Integr. (fit) & $\left(S I_{\mathrm{f}}\right)$ & 0.04 & 1.3 \\
\hline
\end{tabular}

made through moment analysis to take into account the additional uncertainty due to sampling.

These errors do not include systematic errors introduced in the data recording and data reduction. An estimate of these errors can be obtained by a comparison with results from a standard observation at $92 \mathrm{~cm}$ with the WSRT. For this we used a deep $(6 \times 12 \mathrm{~h})$ observation carried out by one of us ( $\mathrm{G}$ de Bruyn) of a field that is not part of the mini-survey and compared this with WENSS data already available for this field. Figure 8 shows the ratio of integrated flux densities as measured by WENSS and the standard WSRT observation. This figure indicates that there are no systematic errors. The figure also shows that the error estimates are reasonable.

Extendedness An estimate of the extendedness of a source can be obtained from the ratio of the integrated flux to the peak flux $S I / S=b_{\mathrm{M}} b_{\mathrm{m}} /\left(B_{\mathrm{M}} B_{\mathrm{m}}\right)$. However, a direct application of Eq. (9) to establish the significance of a result $S I / S>1$ is only possible if the errors $\sigma_{S I}$ and $\sigma_{S}$ are independent. This is not the case. Rather, $S I / S$ shows a very skew distribution, with a tail toward high flux ratios, especially at low signal-to-noise ratios. The median of this distribution is found to be less than 1 .

To establish a criterion for extendedness, we have determined the upper envelopes of the distribution of $S I / S$, 


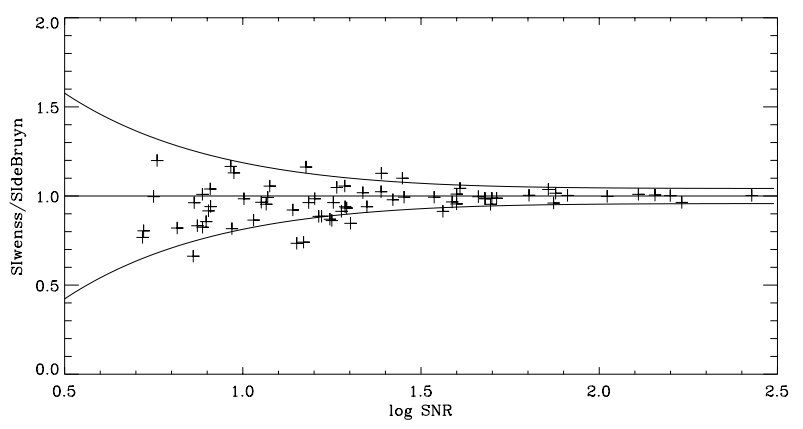

Fig. 8. The ratio of integrated flux densities as measured by WENSS (SIwenss) and a standard WSRT observation (SIdeBruyn), as a function of the signal-to-noise ratio in WENSS. The curves represent $1 \sigma$ errors, under the assumption that the error in the standard WENSS observation is comparable to the error in the WENSS data

containing respectively $80 \%, 90 \%, 95 \%$, and $99 \%$ of the unresolved sources, using Monte-Carlo simulations.

These upper envelopes can be characterized by the equation:

$S I / S=1+\left\{(0.04)^{2}+C^{2}\left(\frac{\sigma_{\mathrm{rms}}}{S}\right)^{2}\right\}^{\frac{1}{2}}$.

The values for $C$ can be found in Table 9 . Thus substituting the value 2.4 for $C$ in this equation gives the ratio $S I_{\mathrm{m}} / S_{\mathrm{m}}$ below which lies $95 \%$ of the unresolved sources with given $S_{\mathrm{m}} / \sigma_{\mathrm{rms}}$.

Table 9. Values for the constant $C$ in Eq. (10), used to determine a criterion for extendedness

\begin{tabular}{lll}
\hline & \multicolumn{2}{c}{$C$} \\
Envelope & moments & fits \\
\hline $80 \%$ & 1.0 & 1.4 \\
$90 \%$ & 1.7 & 2.2 \\
$95 \%$ & 2.4 & 3.2 \\
$99 \%$ & 4.0 & 6.0 \\
\hline
\end{tabular}

Figure 9 shows the distribution the ratio of integrated to peak flux as a function of signal-to-noise ratio. In Fig. 9a the lines show the $90 \%$ and $95 \%$ upper envelopes, used in distinguishing between resolved and unresolved sources. Figures $9 \mathrm{~b}$ and $\mathrm{c}$ show the skew distribution of the flux ratio at two different signal-to-noise ratios. This skewness is a property of the distribution for unresolved sources. However, part of the tail can be ascribed to truly resolved sources.
Morphology The relative errors in the estimates of the flux densities, the major and minor axes $\left(b_{\mathrm{Maj}}, b_{\min }\right)$ and the position angle are not independent. We find, from the Monte-Carlo simulations of unresolved sources, that at low signal-to-noise ratios $(<10)$ the ellipticity of sources is overestimated, resulting in an overestimate of the major axis and an underestimate of the minor axis. Figure 10 shows the median values and the errors found for the major and minor axes as a function of signal-to-noise ratio. The relative error, with respect to the median, can be expressed with the following relation.

$\frac{\sigma_{b}}{b}=\left\{(0.03)^{2}+C\left(\frac{\sigma_{\mathrm{rms}}}{S}\right)^{2}\right\}^{\frac{1}{2}}$

with $C=2.5$ for the major axis, and $C=0.8$ for the minor axis. The lines in Fig. 10 are given by this expression.

Although Eq. (11) has been established from the response of the source finding algorithm to unresolved sources, this expression should give a reasonable approximation of the errors for resolved sources.

\subsection{Completeness and source counts}

The detection of a source with a given intrinsic (noisefree) peak flux density depends on the ratio of the noiseadjusted peak-flux density over the local noise level. A source with an intrinsic peak flux density of $7 \sigma_{\text {rms }}$ will have a noise-adjusted peak density between 5 and $9 \sigma_{\text {rms }}$ in $95 \%$ of the cases (assuming a normal distribution for the noise). From these numbers and from the noise distribution, as shown in Fig. 5, we estimate WENSS to be essentially complete at $30 \mathrm{mJy}$.

The Euclidean normalized differential source counts for the mini-survey are shown in Fig. 11. A comparison with a third degree polynomial parameterization of the source counts for deep WSRT $92 \mathrm{~cm}$ surveys from Wieringa (1991b), shows that WENSS indeed starts to miss sources below approximately $30 \mathrm{mJy}$. The detection rate drops below $50 \%$ already at $25 \mathrm{mJy}$, although the limiting flux density of the survey is approximately $18 \mathrm{mJy}$, given a $5 \sigma_{\text {rms }}$ source in a region where the noise level is 3.5 mJy (less than $20 \%$ of the survey area).

\subsection{Extended sources}

Appendix A shows contour plots of 120 sources in the mini-survey that have a marked extended structure, and a signal-to-noise ratio of the peak of at least 20 . These sources are either resolved single components ("S") sources with a flux-ratio $S I / S>1.5$, or multiple component ("M") sources with one or more resolved components with $S I / S>1.3$.

The contour plots are labeled by the source name, type and flag. Contour levels are $-3.5 \sigma,-2 \sigma, 2 \sigma, 3.5 \sigma, 5 \sigma, 7 \sigma$, $10 \sigma, 15 \sigma, 30 \sigma, 50 \sigma, 100 \sigma, 200 \sigma$, and $500 \sigma$, where $\sigma$ is the local noise level, which can be read from the catalogue. 

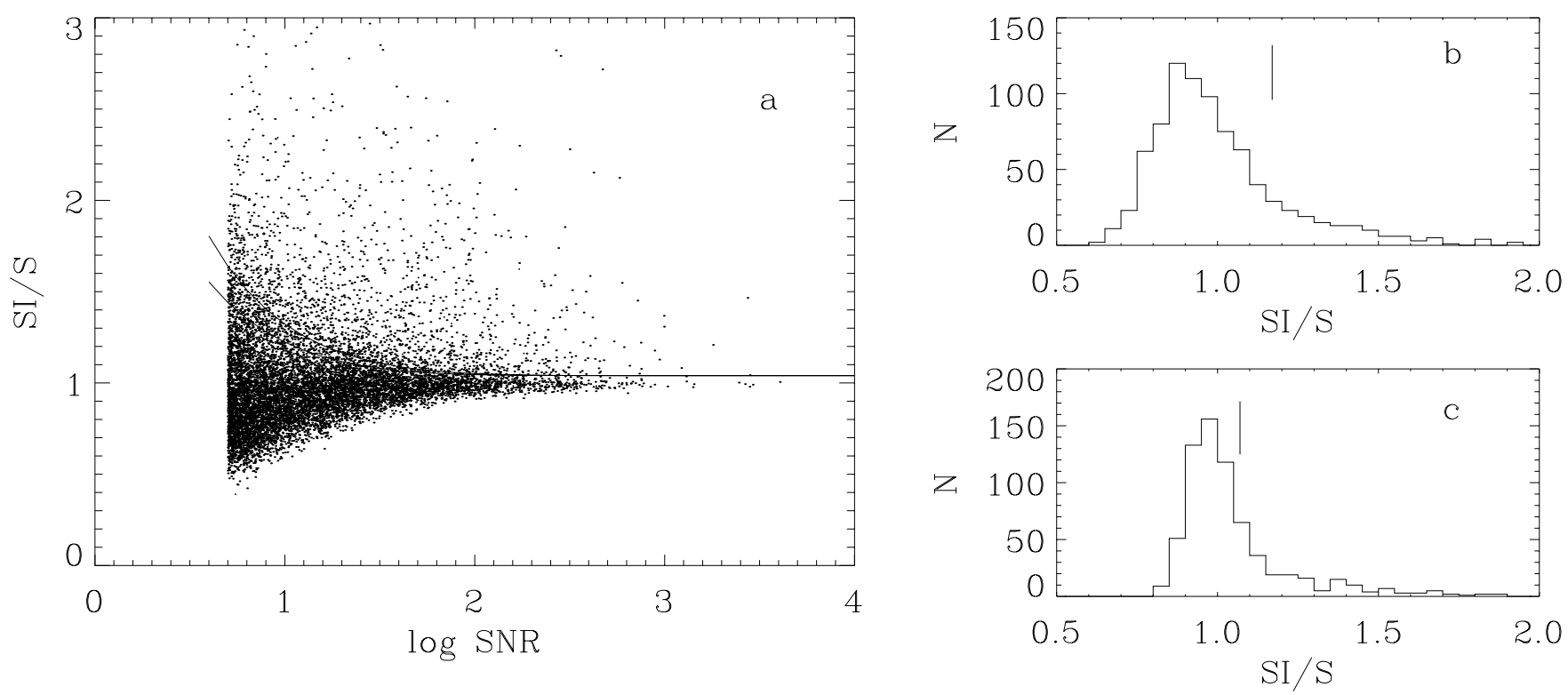

Fig. 9. a) The measured ratio of integrated to peak flux as a function of signal-to-noise ratio for data from the mini-survey. The lines show the upper envelope containing respectively $90 \%$ and $95 \%$ of the unresolved sources, established using Monte-Carlo simulations. b-c) Two distributions of the flux ratio at different signal-to-noise ratios. The vertical lines mark the flux ratio below which one would find $95 \%$ of the unresolved sources. a) $12<\mathrm{SNR}<15$, b) $35<\mathrm{SNR}<50$
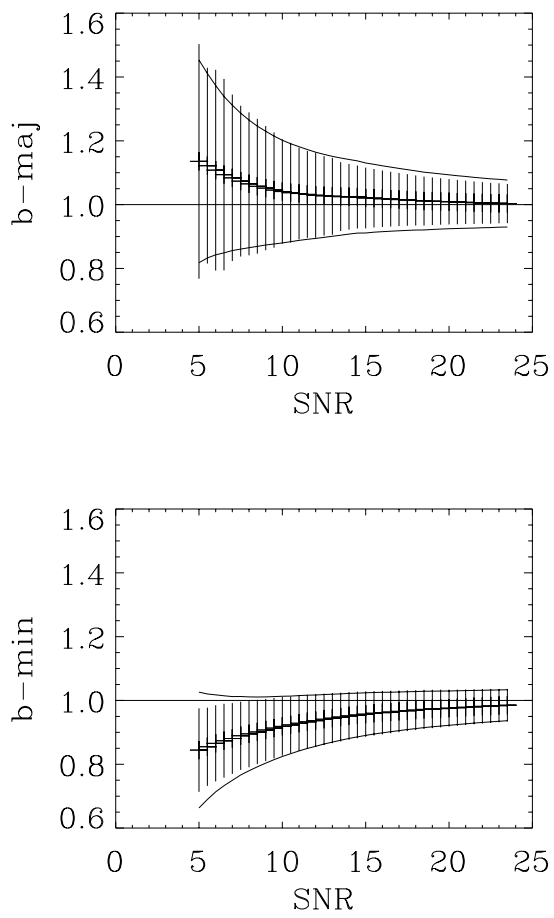

Fig. 10. The median and standard deviation of the major (top) and minor axes (bottom) for unresolved sources as a function of signal-to-noise ratio. The lines represent the error estimate from Eq. (11) with respect to the median. The major and minor axis have been normalized to the beam size

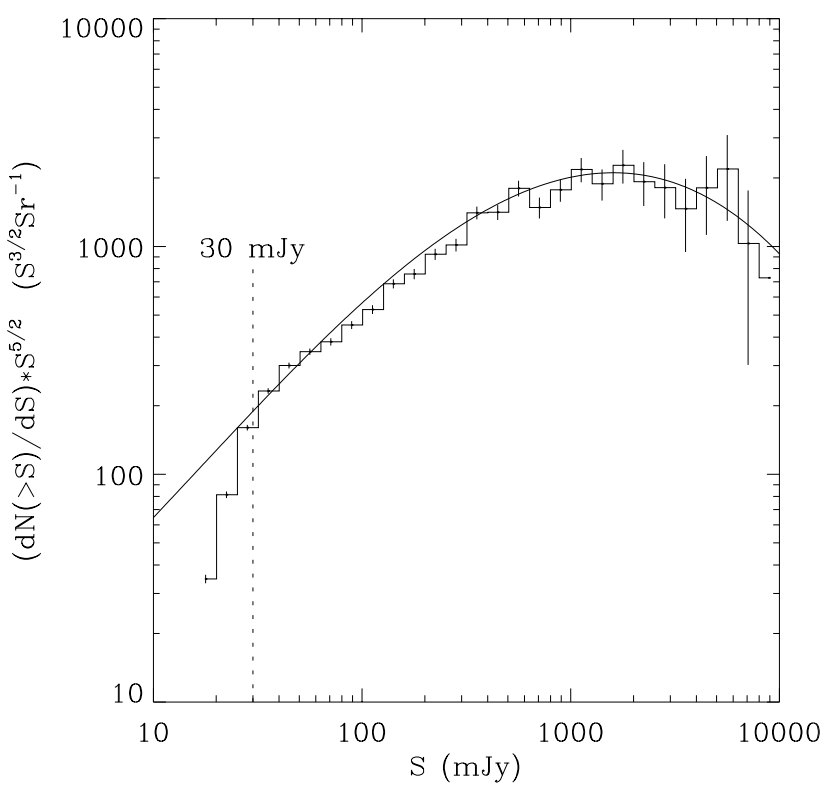

Fig. 11. Euclidean normalized differential source counts for the mini-survey

\section{Conclusions and future plans}

We have presented the first installment of the Westerbork Northern Sky Survey and discussed the uncertainties in the resulting catalogue. Reduction of the full WENSS 
survey should be completed in the summer of 1997. Analysis of the mini-survey has shown that the full WENSS survey will be an important data base for tackling many important astrophysical problems.

A combination of WENSS with existing large sky radio catalogues will produce radio colour-colour and colourmagnitude diagrams over a large area of sky. This will enable us to separate various types of sources to flux-levels fainter by at least an order of magnitude than was previously possible, and should provide new data on the evolution of the space density of distant radio galaxies as a function of spectral index. In addition, WENSS will be used to study large-scale clustering of radio sources taking into account the radio colour discriminant and the optical identification information.

Acknowledgements. We would like to thank Peter Katgert for useful discussions. We acknowledge support from an EU twinning project, funding from the high- $z$ programme subsidy granted by the Netherlands Organization for Scientific Research (NWO), and a NATO research grant. This research was supported by the Netherlands Foundation for Research in Astronomy (NFRA). WENSS is a joint project of the NFRA and Leiden Observatory. The WSRT is operated by the NFRA with financial support from NWO.

\section{Appendice A. Extended sources}

This appendix shows sources from the mini-survey that have a marked extended structure. The contour plots are labeled by the source name, type and flag. Contour levels are $-3.5 \sigma,-2 \sigma, 2 \sigma, 3.5 \sigma, 5 \sigma, 7 \sigma, 10 \sigma, 15 \sigma, 30 \sigma, 50 \sigma$, $100 \sigma, 200 \sigma$, and $500 \sigma$, where $\sigma$ is the local noise level, which can be read from the catalogue.

\section{References}

Becker R.H., White R.L., Edwards A.L., 1991, ApJ 75, 1 Becker R.H., White R.L., Helfland D.J., 1995, ApJ 450, 559 Böhringer H., Voges W., Ebeling H., et al., 1991, in Shanks
T., Banday A., Ellis R. and Frenk C. (eds.) Observational Tests of Cosmic Inflation, p. 210

Bower R.G., Hasinger G., Castander F.J., et al., 1996, MNRAS 281, 59

Condon J., Broderick J., Seielstad, G., 1989, AJ 97, 1064

Condon J.J., 1996, Errors in elliptical Gaussian fits (preprint)

Condon J.J., Cotton W.D., Greisen E.W., Perley R.A., Yin Q.F., Broderick J.J., 1993, BAAS 183, 6402

Gregory P.C., Condon J.J., 1991, ApJS 75, 1011

Gregory P.C., Scott W.K., Douglas K., Condon J.J., 1996, ApJS 103, 427

Hacking P., Houck J.R., 1987, ApJS 63, 311

Hales S.E.G., Baldwin J.E., Warner P.J., 1993, MNRAS 263, 25

Högbom J.A., 1974, A\&AS 15, 417

Irwin M., Maddox S., McMahon R., 1994, Spectrum, newsletter of the Royal Observatories 2, 14

Kaper H.G., Smiths D.W., Schwartz U., Takakubo K., van Woerden H., 1966, BAN 18, 465

Kolkman O.M., 1993, The Westerbork Synthesis Radio Telescope User Documentation, NFRA

Lacy M., Rawlings S., Warner P.J., 1992, MNRAS 256, 404

Lacy M., Riley J.M., Waldram E.M., McMahon R., Warner P., 1995, MNRAS 614, 276

McGilchrist M.M., Baldwin J.E., Riley J.M., et al., 1990, MNRAS 246, 110

Myers S.T., Fassnacht C.D., Djorgovski S.G., et al., 1995, ApJL 447, L5

Patnaik A.R., Browne I.W.A., Wilkinson P.N., Wrobel J.M., 1992, MNRAS 254, 655

Press W.H., Flannery B.P., Teukolsky S.A., Vetterling W.T., 1992, Numerical Recipes in C, 2nd ed.. Cambridge University Press

Rees N., 1990, MNRAS 244, 233

Snellen I.A.G., de Bruyn A.G., Schilizzi R.T., Miley G.K., Myers S.T., 1995, ApJL 447, L9

Visser A.E., Riley J.M., Röttgering H.J.A., Waldram E.M., 1995, A\&AS 110, 419

Wieringa M., 1991a, in IAU 131: Radio interferometry: Theory, techniques, and applications, p. 192

Wieringa M., 1991b, Ph.D. Thesis, Leiden University 

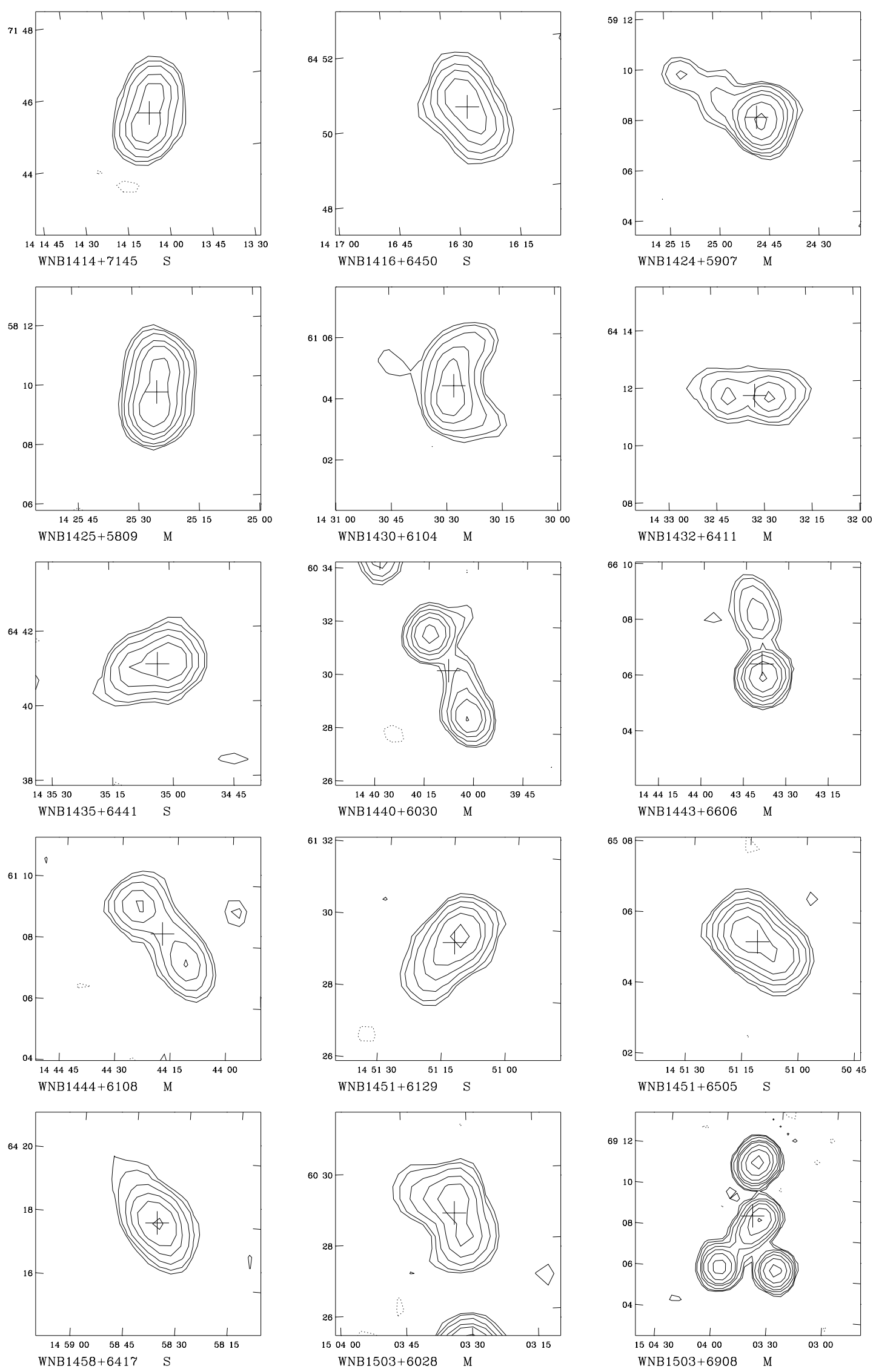

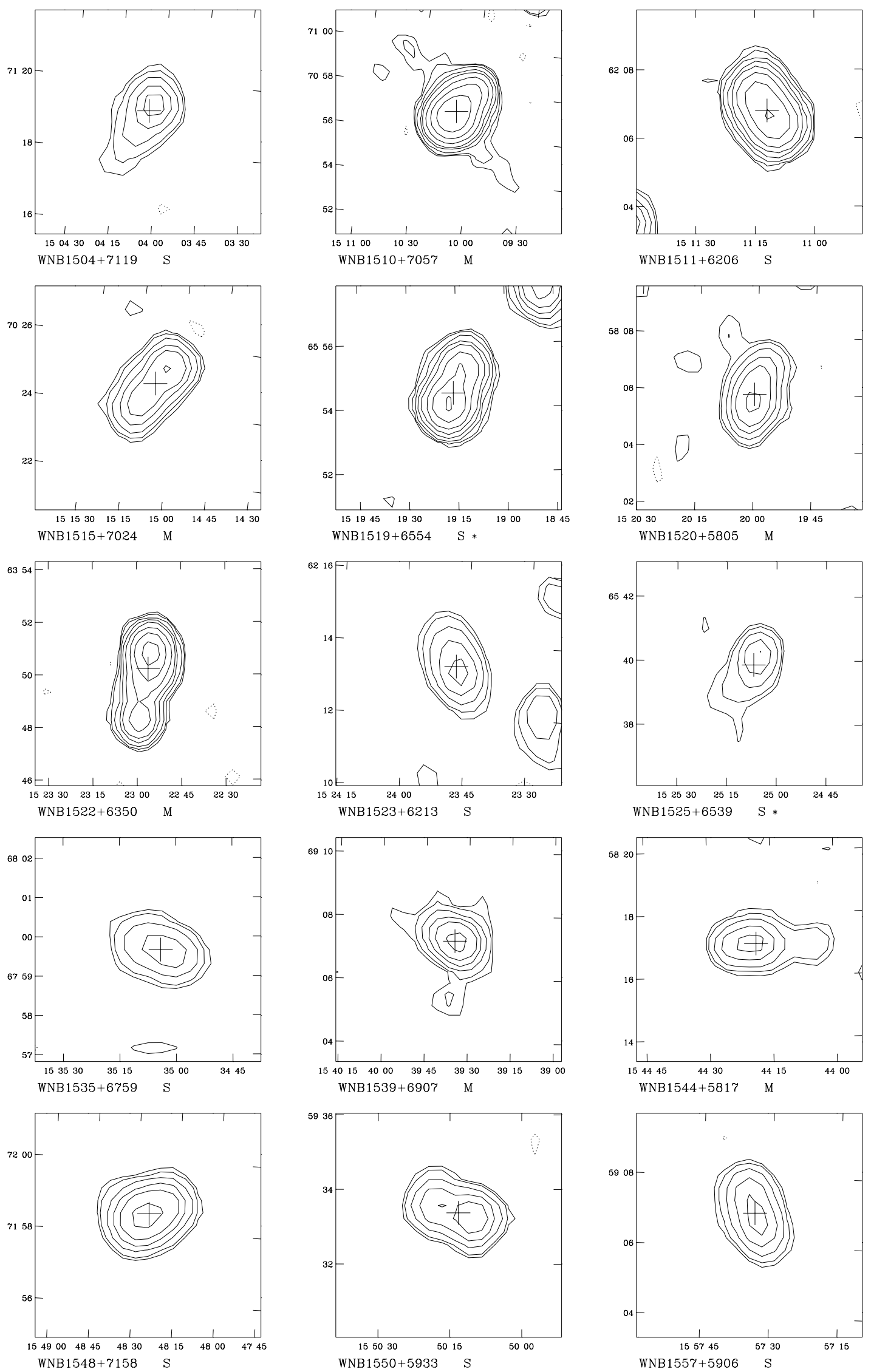

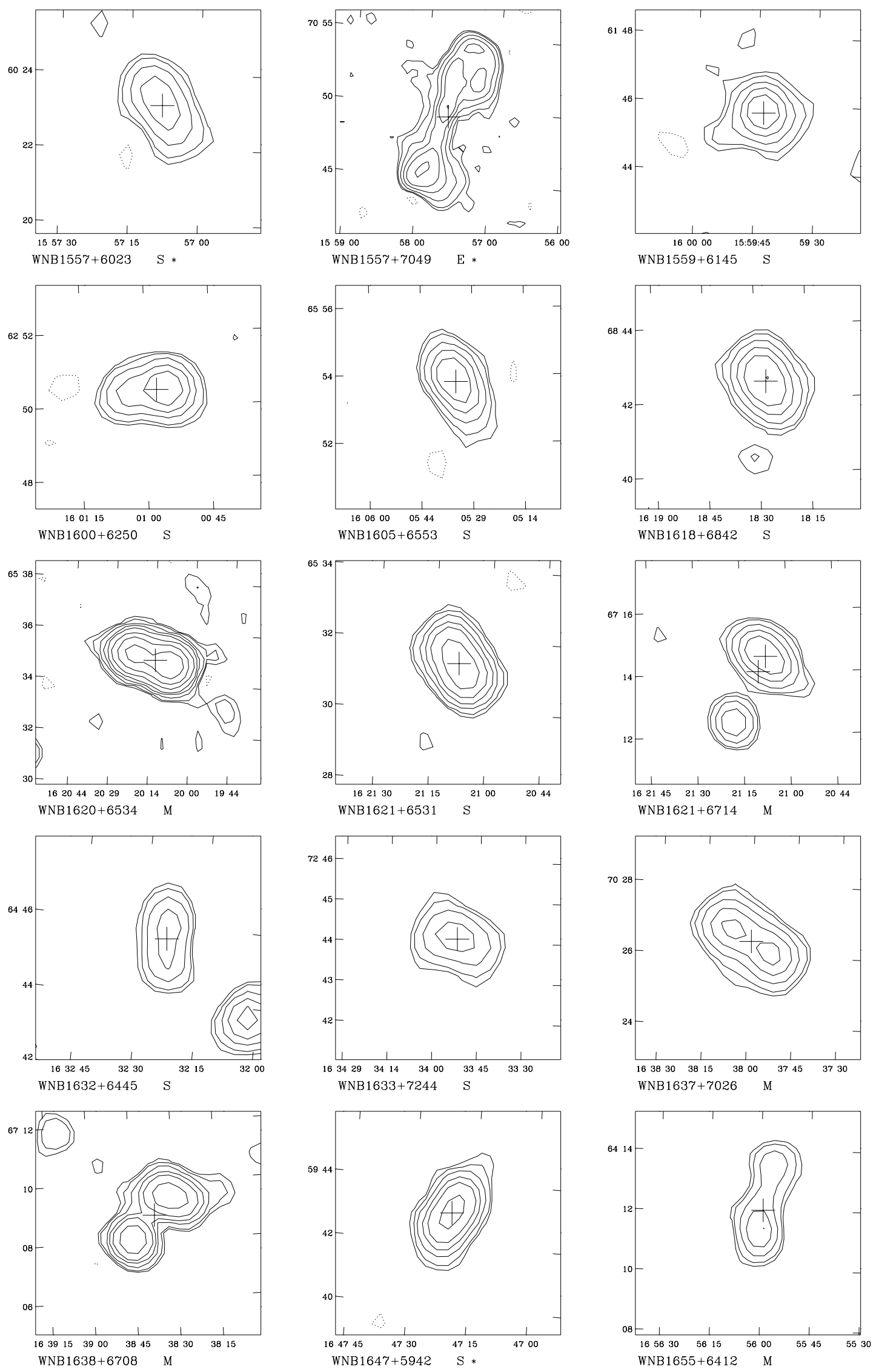

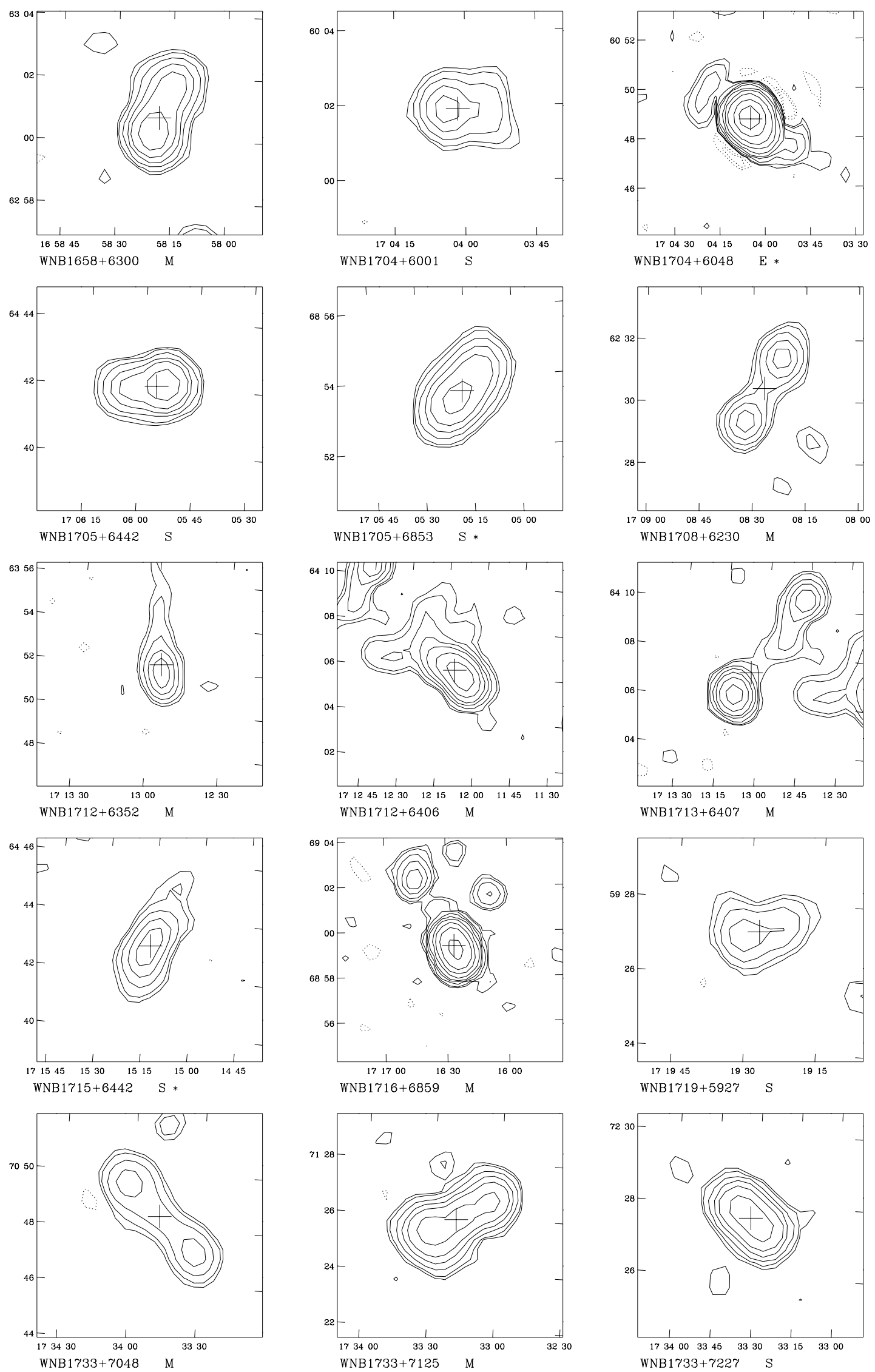

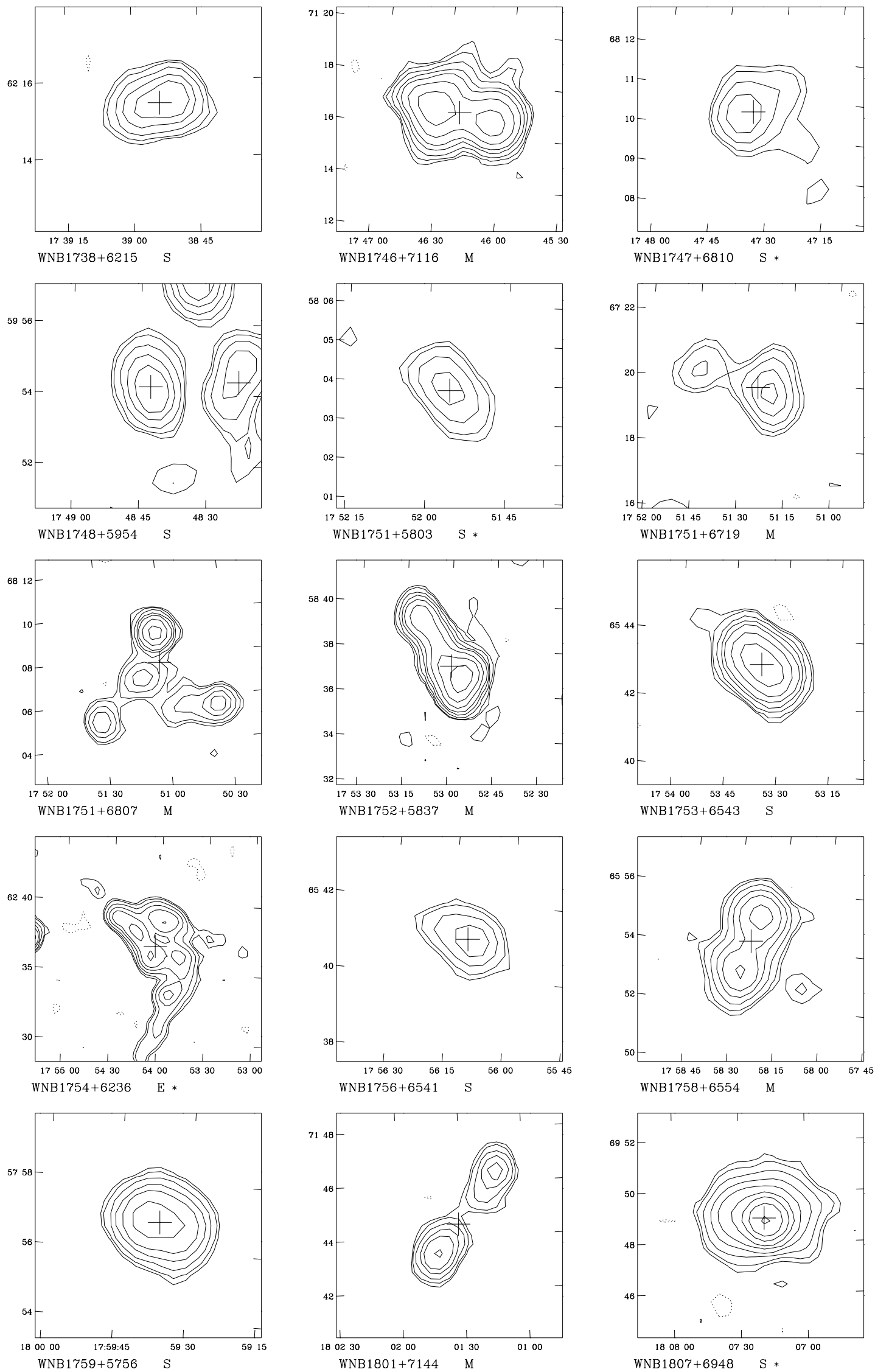

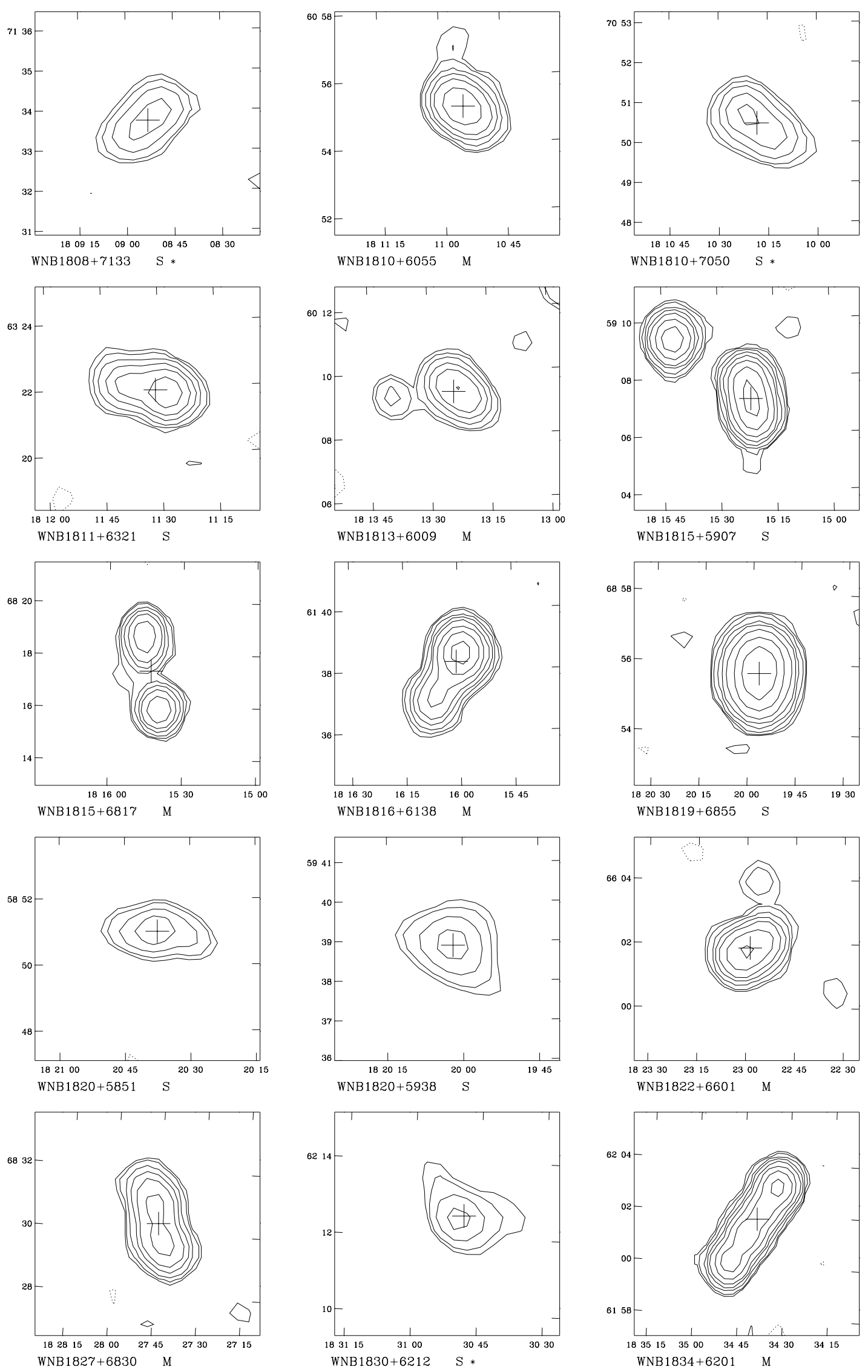

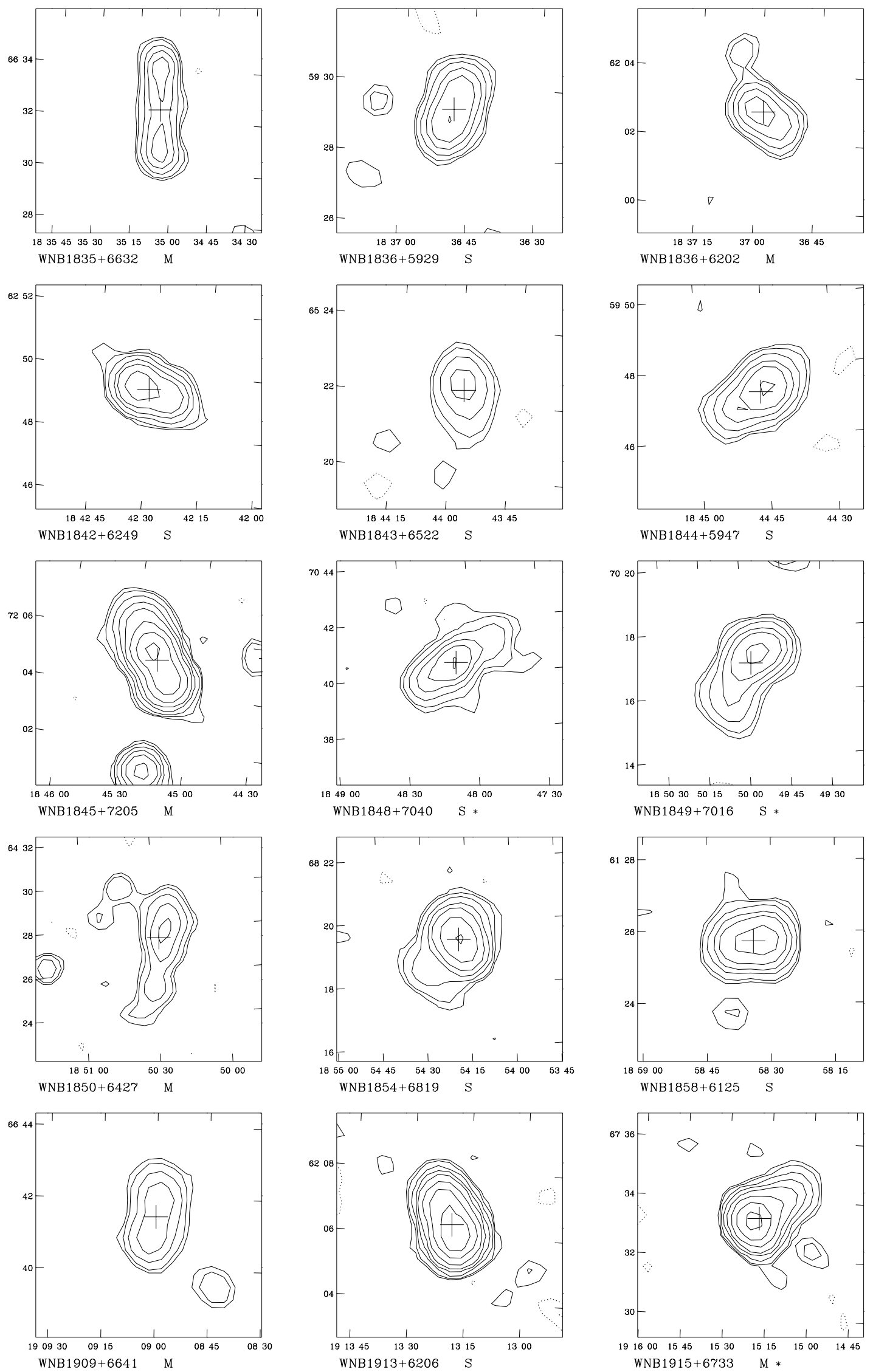

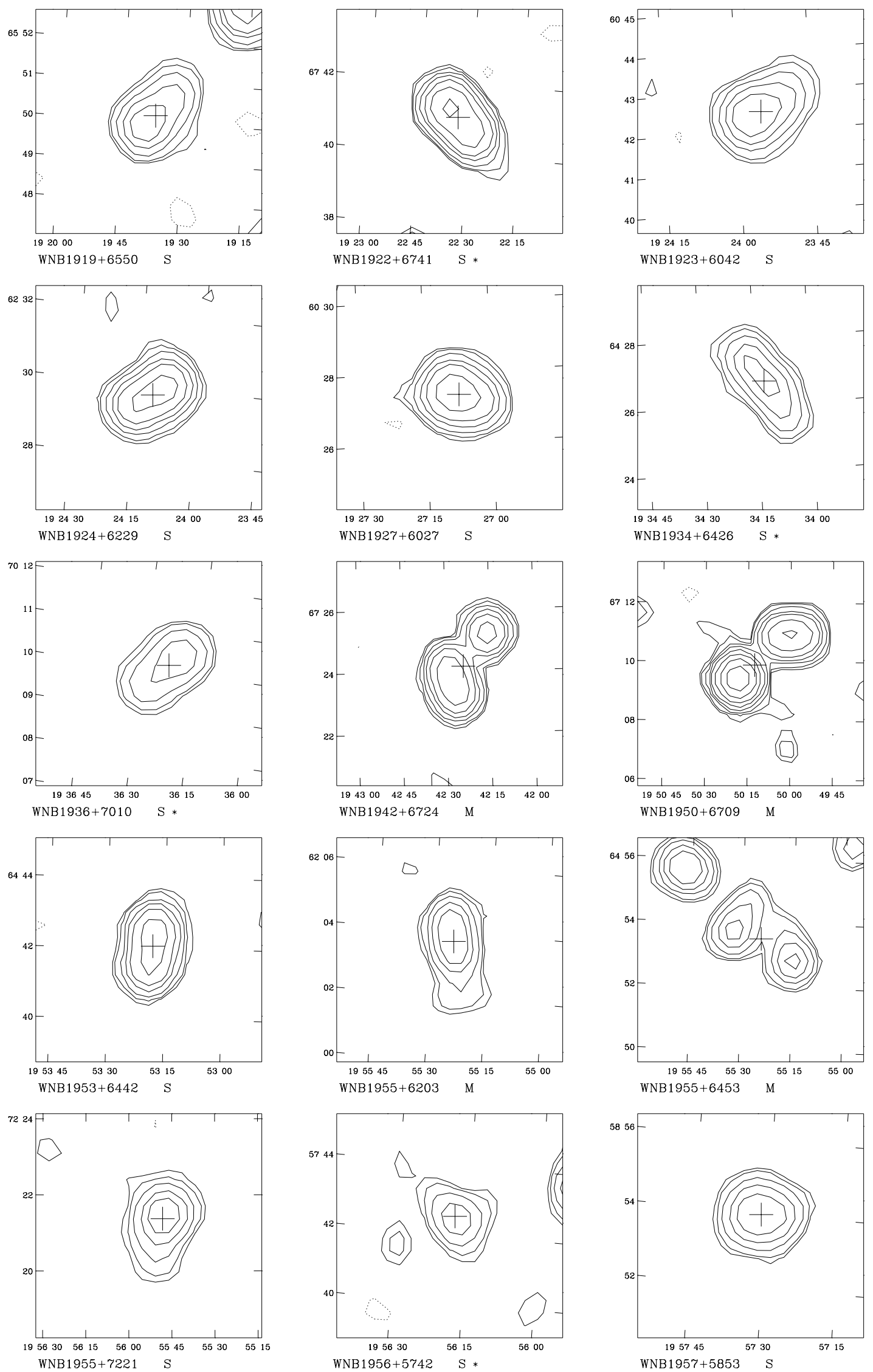

WNB1956+5742 S*

WNB1957+5853 